\title{
Catalytic Nanotruss Structures Realized by Magnetic Self-Assembly in Pulsed Plasma
}

Sebastian Ekeroth, Peter Münger, Robert Boyd, Joakim Ekspong, Thomas Wågberg, Ludvig Edman, Nils Brenning and Ulf Helmersson

The self-archived postprint version of this journal article is available at Linköping University Institutional Repository (DiVA):

http://urn.kb.se/resolve?urn=urn:nbn:se:liu:diva-148107

N.B.: When citing this work, cite the original publication.

Ekeroth, S., Münger, P., Boyd, R., Ekspong, J., Wågberg, T., Edman, L., Brenning, N., Helmersson, U., (2018), Catalytic Nanotruss Structures Realized by Magnetic Self-Assembly in Pulsed Plasma, Nano letters (Print), 18(5), 3132-3137. https://doi.org/10.1021/acs.nanolett.8bo0718

Original publication available at:

https://doi.org/10.1021/acs.nanolett.8boo718

Copyright: American Chemical Society

http://pubs.acs.org/ 


\title{
Catalytic nanotruss structures realized by magnetic
}

\section{self-assembly in pulsed plasma}

\author{
Sebastian Ekeroth ${ }^{1}$, E. Peter Münger ${ }^{1}$, Robert Boyd ${ }^{1}$, Joakim Ekspong ${ }^{2}$, Thomas Wågberg ${ }^{2}$, \\ Ludvig Edman', Nils Brenning ${ }^{1,3}$, Ulf Helmersson ${ }^{1}$ \\ 1) Linköping University, Department of Physics, SE-581 83 Linköping, Sweden. \\ 2) Umeå University, Department of Physics, SE-901 87 Umeå, Sweden.
}

${ }^{3)}$ KTH Royal Institute of Technology, School of Electrical Engineering, Division of Space and Plasma Physics, SE-100 44 Stockholm, Sweden.

Tunable nanostructures that feature high surface area, are firmly attached to a conducting substrate and can be fabricated efficiently over significant areas, are of interest for a wide variety of applications in, for instance, energy-storage and catalysis. We present a novel approach to fabricate Fe nanoparticles using a pulsed plasma process and their subsequent guidance and self-organization into well-defined nanostructures on a substrate of choice by the use of an external magnetic field. A systematic analysis and study of the growth procedure demonstrate that non-desired nanoparticle agglomeration in the plasma phase is hindered by electrostatic repulsion, that a polydisperse nanoparticle distribution is a consequence of the magnetic collection, and that the formation of 
highly networked nanotruss structures is a direct result of the polydisperse nanoparticle distribution. The nanoparticles in the nanotruss are strongly connected and their outer surfaces are covered with a 2-nm layer of iron oxide. A 10- $\mu \mathrm{m}$ thick nanotruss structure was grown on a lightweight, flexible and conducting carbon-paper substrate, which enabled the efficient production of $\mathrm{H}_{2}$ gas from water splitting at a low overpotential of $210 \mathrm{mV}$ and at a current density of $10 \mathrm{~mA} / \mathrm{cm}^{2}$.

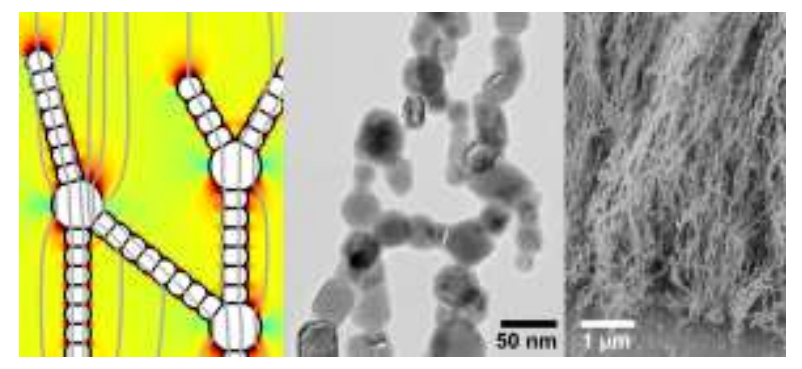

Keywords: nanotrusses, nanowires, nanoparticles, iron, electrocatalysis, pulsed sputtering

Solution-based magnetic self-assembly of in situ or ex situ synthesized ferromagnetic nanoparticles is currently a vibrant field ${ }^{1-9}$, partially since the resulting nanostructures promise important or improved application in energy storage ${ }^{10,11}$, chemical production ${ }^{12}$, and ultra-highdensity magnetic storage ${ }^{13}$. A drawback with this solution-based approach is the difficulty in keeping the nanoparticles clean from oxides and other impurities, and that the inter-particle contacts and resulting nanostructures consequently suffer in both stability and electrical conductivity. Magnetic nanoparticles can also be grown by the method of arc discharge plasma, but although creative attempts have been made to reduce the amount of carbon impurities by inclusion of an external magnetic field ${ }^{14}$, significant amounts of carbon impurities are typically still present.

An alternative method for nanoparticle synthesis is by plasma sputtering. The employment of a controlled atmosphere results in a high degree of impurity control, however, the main setback with 
conventional continuous-plasma nanoparticle deposition is inefficient production ${ }^{15}$. The recent invention of pulsed-plasma deposition improves the yield by up to two orders of magnitude compared to continuous-plasma deposition ${ }^{16,17}$.

Here, we combine pulsed-plasma fabrication of nanoparticles with magnetic-self-assembly within the same reaction chamber, and demonstrate that this novel approach results in an efficient production of ordered and stable ferromagnetic nanostructures on a substrate of choice. Specifically, we show that branched wires of Fe nanoparticles can be fabricated onto both metal and carbon paper substrates, and that the nanostructure stability is due to both strong particleparticle bonding and a large degree of interlinking between the nanowires through branching.
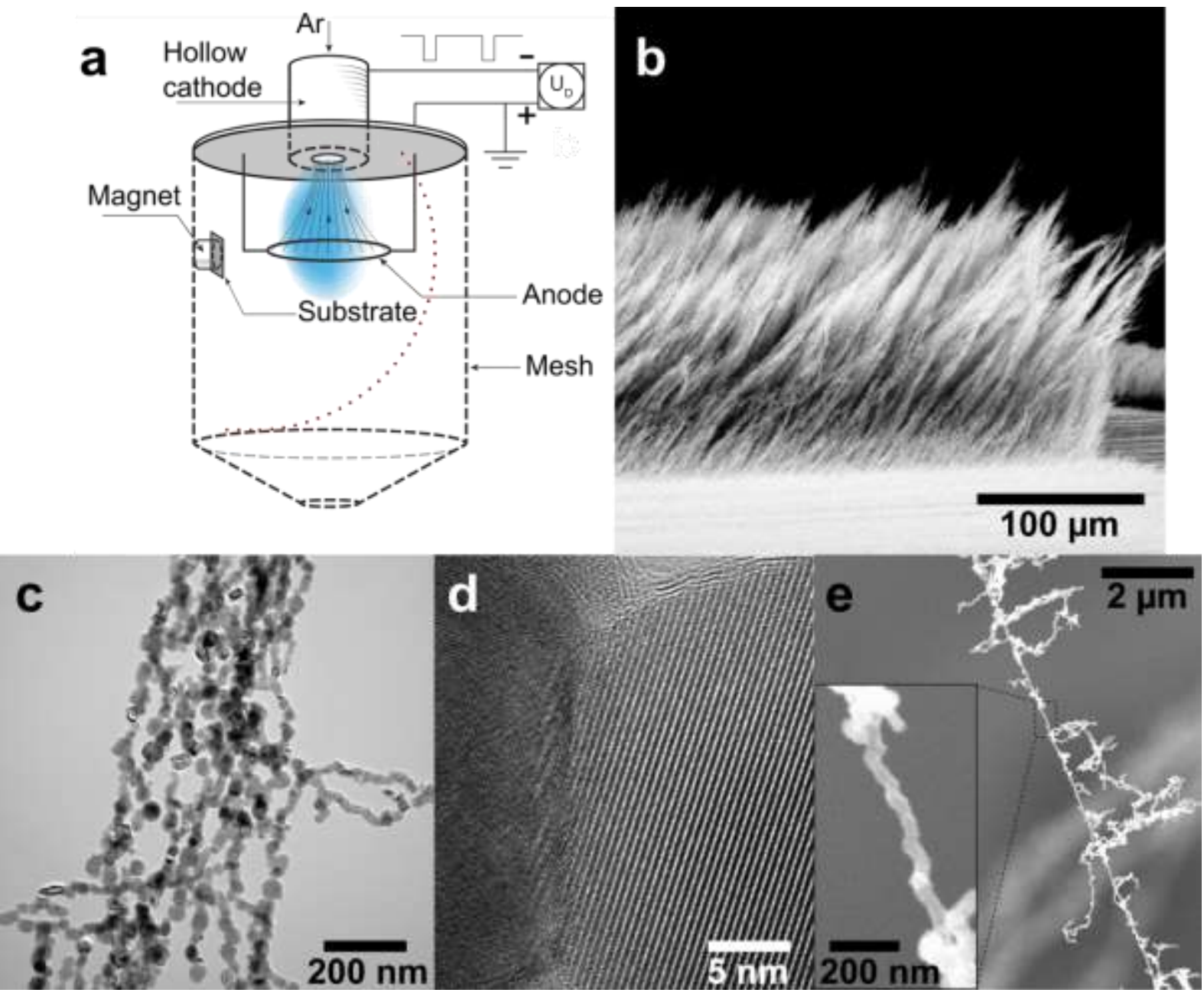

Figure 1. Experimental setup and generated nanostructures. (a) Schematic of the pulsed-plasma 
fabrication setup. The blue shaded area represents the high electron-temperature region and the dotted red line indicates the outer edge of the magnetic capture zone for nanoparticles with a diameter of $40 \mathrm{~nm}$. (b) SEM image of a dense grass-like array oriented by an external magnet. (c) TEM image displaying the truss-like architecture of individual strains in this array. (d) TEM image of the contact between two nanoparticles. (e) SEM image of a stretched nanowire. The highermagnification image in the lower left inset indicates a plastic deformation of the nanoparticles during stretching.

The fabrication setup is schematically shown in Fig. 1a. It essentially comprises a hollow Fe cathode, through which Ar gas is fed into the reaction chamber, and below which a grounded stainless-steel ring anode is positioned. By applying high-power electric pulses to the cathode, Fe atoms are sputtered out from the inside of the cathode, and when they enter the intense plasma region they become ionized. A cylindrical mesh, kept at floating potential, is confining the plasma. $\mathrm{O}_{2}$ gas was also introduced into the chamber, but at a much lower flow rate. This is an established technique to reduce variations in the nanoparticle production rate. ${ }^{18}$ In our process, the introduction of $\mathrm{O}_{2}$ gas implies that a two-body collision in the form of $\mathrm{O}_{2}+\mathrm{Fe} \rightarrow \mathrm{FeO}+\mathrm{O}$ becomes dominant over the slower three-body collision of $2 \mathrm{Fe}+\mathrm{Ar} \rightarrow \mathrm{Fe}_{2}+\mathrm{Ar}$ for the first step of nucleation (the $\mathrm{Ar}$ atom is a critical constituent in the latter reaction, since it carries away the excess energy required to keep the $\mathrm{Fe}_{2}$ dimer bound). Furthermore, the $\mathrm{Fe}$ in an oxidized cluster is stronger bound than an Fe in a pure metal cluster, which reduces the evaporation rate during growth and makes the critical size for stable clusters smaller. However, it is notable that the required $\mathrm{O}_{2}$ flow rate was so low that the overall oxidation of the nanoparticles is negligible.

The substrate on which the nanoparticles are collected, and the nanostructures are grown, is positioned at the inner edge of the mesh at the same height as the ring anode. A permanent magnet 
is placed behind the substrate to effectively guide the ferromagnetic nanoparticles to the substrate. The process can be upscaled, e.g. by using hollow cathode arrays or linear hollow cathodes. More experimental details are available in the Methods Section.

Fig. 1b presents a scanning electron microscopy (SEM) image of the material collected on a Ticoated $\mathrm{Si}$-substrate, following 10 min exposure to the plasma and with a $0.36 \mathrm{~T}$ permanent magnet positioned behind the substrate. A dense and well-oriented grass-like array, with a thickness of 150-200 $\mu \mathrm{m}$, has formed on the substrate. The array invariably orients itself in the direction of the field of the permanent magnet. The transmission electron microscopy (TEM) image in Fig. 1c reveals that the grass-like structure is built up from nanoparticles, with a typical diameter of 10-60 $\mathrm{nm}$, that have assembled into long nanowires with a high degree of branching and linking between the nanowires. A deeper analysis of the structure can be found in the caption of Fig. S1.

High-resolution TEM imaging (for details see Supporting Information Fig. S2) reveals that: (i) the interior bulk of the nanoparticles comprises pure Fe crystallized in a single domain in the bccphase, $\alpha$-Fe; (ii) the outer nanowire surface exposed to the ambient consists of $\mathrm{a} \approx 2 \mathrm{~nm}$ thick crystalline oxide layer, with a lattice spacing compatible with the $\mathrm{Fe}_{3} \mathrm{O}_{4}$ structure. The oxide formation is consistent with the Cabrera-Mott model, which Krajewski et al. used when they reported that a pure iron surface exposed to an oxygen-containing atmosphere will form a $1 \mathrm{~nm}$ thick iron oxide layer within $0.2 \mathrm{fs}$, a $2 \mathrm{~nm}$ layer within $40 \mathrm{~s}$, and a $3 \mathrm{~nm}$ layer within 40 weeks ${ }^{7}$. Fig. 1d is a TEM image of a typical nanoparticle-nanoparticle contact area. The vertical straight interface between the two Fe nanoparticles displays a Moiré pattern, which is a strong indicator of a well-ordered interface free of oxides. It should be pointed out, although most particle-particle contacts appear to be free of oxides, not all are. A few observations clearly show a thin oxide layer in-between the particles. The origin of this interfacial oxide is unclear, but could be due to partial oxidation of the nanoparticle surface before assembly or due to oxidation of the metal-metal 
interface after removal from the vacuum system by diffusion of oxygen in the grain boundary between the nanoparticles.

The relative large contact surfaces between the irregularly formed nanoparticles suggest reshaping of the nanoparticles after they have come in contact. This can be explained by pressure and temperature induced sintering. The magnetic force between two cubic $40 \mathrm{~nm}$ single-domain magnetized nanoparticles in contact, independent of any external magnetic field, gives a pressure of $3 \mathrm{MPa}$ for a contact area of $30 \times 30 \mathrm{~nm}^{2}$ and proportionally higher for smaller contact areas. It is difficult to estimate the actual temperature of the nanoparticles in our process, but Magnolini and Kortshagen have reported a temperature of several hundred $\mathrm{K}$ above the gas temperature in a plasma system. ${ }^{19}$ Sintering of Ag nanoparticles have been demonstrated by Guo et al. at $3 \mathrm{MPa}$ and $523 \mathrm{~K}$. For Ag $523 \mathrm{~K}$ corresponds to $34 \%$ of the melting temperature. ${ }^{20}$ For Fe the corresponding temperature is $616 \mathrm{~K}$ suggesting that sintering between the Fe nanoparticles is likely.

By removing the magnet, nanostructures featuring a random orientation and without significant branching and linking were fabricated, as shown in Fig. S3. This procedure allowed for facile extraction and studies of single wires, and one such single wire is shown in the SEM micrograph in Fig. 1e. One should note here that all nanowires grown with the present technique are wiggly and never straight, probably due to irregularly shaped nanoparticles. The wire in Fig. 1e, that comprises $\approx 200$ nanoparticles in the field of view, is unintentionally stretched during preparation for microscopy to a straight line without breaking. This implies a strong inter-particle bond also in the case where no external magnetic field is applied. This is further supported by high resolution TEM (see Fig. S4) illustrating metal-metal bonded nanoparticles. To further illustrate the strength of the two types of growth structures (with and without external magnet), samples of the two were put through ultrasonic treatment. Both kept their nanowires intact, but for the samples grown 
without an external magnet it was possible to separate different nanowires from each other. TEM images of these samples can be seen in Fig. S5.

With the hierarchical nanostructure being characterized, we turn our attention to the understanding and elucidation of the nanostructure formation process within the growth chamber. A first question relates to whether the nanoparticles are beginning to assemble into nanowires already in the plasma phase, or if this is solely taking place at, or next to, the substrate (see Fig. 1a). The Fe-based nanoparticles are ferromagnetic, and will as such experience an attractive interparticle force when magnetically aligned. At the same time, the negative charge of the nanoparticles in the plasma ${ }^{17}$ results in a counteracting repulsive electrostatic force.
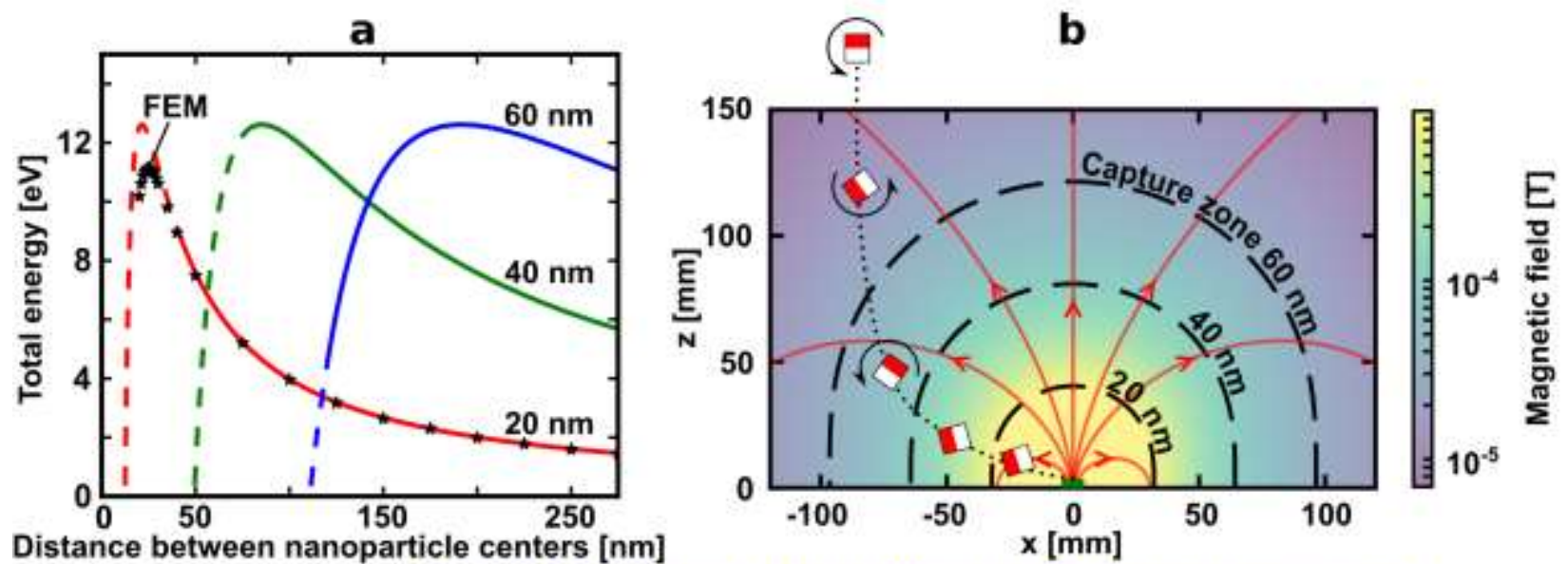

Distance between nanoparticle centers [nm]
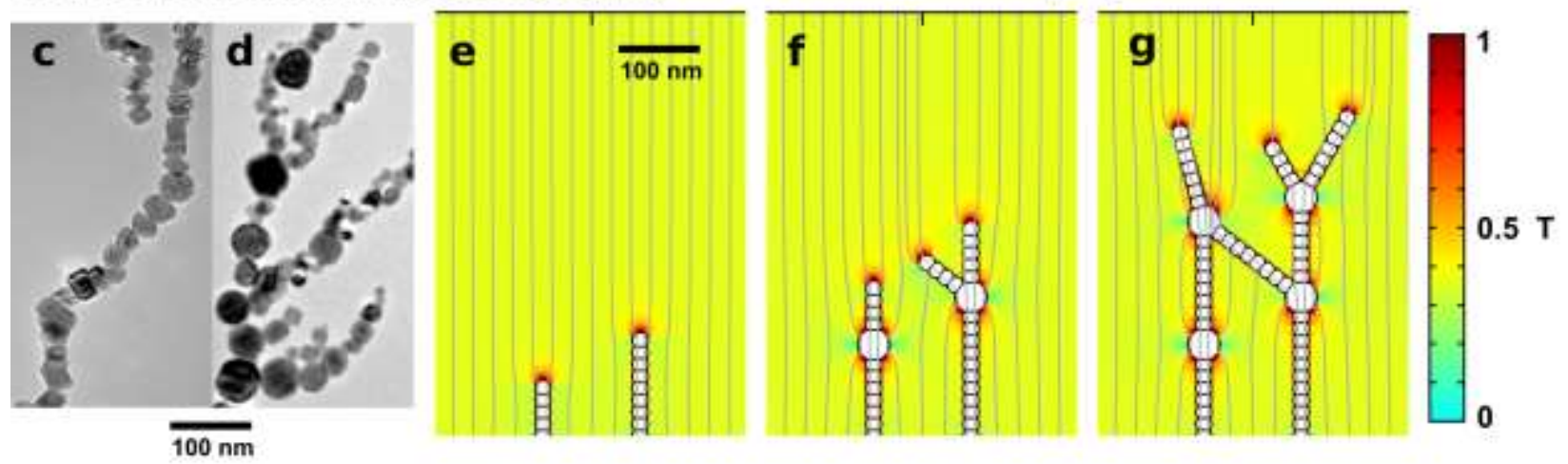

Figure 2. The magnetic influence on the capture and growth processes. (a) The energy barrier against agglomeration of differently sized nanoparticles in a plasma with an electron temperature 
of $1.0 \mathrm{eV}$. The black stars show Finite-Element-Method (FEM) calculations. Dashed lines indicate separations where the analytic model is less good. (b) The magnetic capture zones for fully magnetized $\mathrm{Fe}$ nanoparticles as a function of their diameter, for a $0.36 \mathrm{~T}$ magnet and a kinetic temperature of $300 \mathrm{~K}$. The free rotation and subsequent alignment, of a $40 \mathrm{~nm}$ nanoparticle at the capture zone edge, are depicted by the dotted black line. (c) TEM image of a single linear nanowire comprising narrow size-dispersed nanoparticles. (d) TEM image of a single branched nanowire comprising broad size-dispersed nanoparticles. (e-g) Illustrations of the role of differently sized nanoparticles. Branching and interlinking is promoted by leak fields from the larger nanoparticles.

With this in mind, we have derived an analytic model for the energy barrier against agglomeration. Key results are presented in Fig. 2a and the detailed derivation can be found in the Supporting Information. We find that the height of the energy barrier is strongly dependent on the electron temperature (Fig. S6) but, somewhat surprisingly, independent of the nanoparticle size. A typical value for the electron temperature within this type of plasma is $1 \mathrm{eV}^{17}$, and it translates into an energy barrier of more than $10 \mathrm{eV}$. As the average thermal translational energy of a nanoparticle is only $0.04 \mathrm{eV}$ (at a gas temperature of $300 \mathrm{~K}$ ), this implies that it is effectively impossible for the ferromagnetic nanoparticles to overcome the electrostatic barrier and agglomerate within the plasma. Although the electron temperature will drop closer to the substrate, it is not probable that it in any location in the plasma chamber will reach the calculated electron temperature threshold of $0.15 \mathrm{eV}$ (that results in a barrier height of $0.04 \mathrm{eV}$ ), at which aggregation becomes significant. We thus draw the conclusion that the plasma environment is effective in hindering premature agglomeration of the ferromagnetic nanoparticles.

When a magnet is introduced at the substrate position (see Fig. 1a), the magnetic nanoparticles will tend to align with the external magnetic field and accelerate towards the substrate/magnet. 
This will significantly increase the collection yield of nanoparticles on the substrate, but has also other interesting implications. By analyzing how the magnetic forces on nanoparticles depend on their size and orientation (for details, see Supporting Information), we find the critical distance from the magnet where the nanoparticles are likely to be magnetically aligned and trapped. Fig. $2 b$ illustrates the calculated capture zone boundaries for three differently sized nanoparticles as dashed lines $($ diameter $=20,40,60 \mathrm{~nm})$. A trajectory for a magnetic nanoparticle with a $40 \mathrm{~nm}$ diameter is illustrated in Fig. $2 b$ by the dotted line. Note that the nanoparticle is attracted in the direction $\operatorname{grad}|\mathbf{B}|$ of increasing field strength, which is perpendicular to the capture zone boundaries. The kinetic energy gained by a nanoparticle can help it penetrate opposing electric fields at the substrate.

The fact that the boundary of the capture zone depends on the nanoparticle size has a significant influence on the nanoparticle size distribution on the substrate when an external magnet is employed. For some particles the nucleation takes place close to the substrate, and they are effectively drawn to the magnet when they still are relatively small; but when the nucleation takes place further away, the nanoparticles have to grow larger in size before they are attracted by the magnet. The consequence is thus that the inclusion of the magnet will not only significantly increase the yield, but also result in a polydisperse nanoparticle distribution on the substrate. This predicted behavior has been verified in experiments. Fig. 2c presents a TEM image of the monodisperse nanoparticle distribution that results when the magnet is removed, while the TEM in Fig. 2d displays the polydisperse distribution observed when the external magnet is used.

An additional interesting observation is that the monodisperse nanoparticles invariably arrange into linear wires (Fig. 2c), whereas the polydisperse nanoparticles form networked nanotruss structures (Fig. 2d and Fig. 1c). The physical reason for this difference is revealed in magneticfield simulations. Fig. 2e-g present three stages in the growth of two nanowires on a substrate with 
the magnetic field strength outside the nanoparticles color-coded. It is energetically preferable for a ferromagnetic nanoparticle to dock at a site on the substrate with a large magnetic leak field in order to lower the total magnetic energy of the system. In Fig. 2e, only monodisperse nanoparticles have arrived at the substrate, and for this configuration the largest leak field is localized at the wire end of the growing nanowire, resulting in a linear growth for the two nanowires. In Fig. 2f, we also include two larger nanoparticles, with the consequence that significant leak fields remain when smaller nanoparticles have attached on top of the larger nanoparticles. These remaining leak fields will function as additional connection points for later arriving nanoparticles, and as such allow for branching and nanotruss formation, as depicted in Fig. $2 \mathrm{~g}$. If the supply of nanoparticles continues, this process will go on until the magnetic leak fields are minimized in an interlinked structure with few open ends. Note that, regardless the nanoparticle size, an arriving particle will align itself to assemble north-to-south with the already deposited wire, to minimize the magnetostatic energy.

We now turn our attention to the application potential of the realized nanostructures. In this context, we note with interest that the nanotruss structures appear to feature a manifold of desirable characteristics for efficient catalysis: (i) the nanoparticle surface comprises iron-oxide that is frequently reported to be catalytically active ${ }^{21}$, (ii) the high surface-to-volume ratio allows for a manifold of reactant sites, and the strong inter-particle contacts provides for (iii) mechanical strength and also (iv) for an efficient transport path to the current-distributing substrate.

Fig. 3a presents a photograph of a $40 \times 20 \mathrm{~mm}^{2}$ carbon-paper substrate. Details on its fabrication are available in the Methods section. The carbon fibres comprise a wire network that is visible as the bright features with an average diameter of $10 \mu \mathrm{m}$ in the low-magnification top-view SEM image in Fig. 3b. It appears that this carbon-fibre network is effectively coated by a dense mat of nanotrusses, which is confirmed in the higher-magnification side-view SEM image in Fig. 3c. For 
this particular experiment, the deposition time in the plasma chamber was $5 \mathrm{~min}$, and it resulted in a thickness of the nanotruss deposit on the carbon paper of $\sim 10 \mu \mathrm{m}$.

The electrocatalytic capacity of the nanotruss-coated carbon-paper electrode was investigated by linear sweep voltammetry (LSV) in alkaline water solution; for experimental details see Methods section. When driving such an experiment to a negative potential with respect to the relative hydrogen electrode (RHE), it is possible to split water into hydrogen gas. Fig. 3d presents the LSV data for a bare carbon paper (solid line), a carbon paper coated by nanotrusses for 1/5/10 min (dashed lines), and a high-performance Pt-wire reference (dotted line). The bare carbon paper is essentially inactive over the entire potential range, which demonstrates that it is not catalytically active for water splitting. The nanotrusses are in contrast highly active, and the 10-min-nanotruss electrode only required an overpotential of $210 \mathrm{mV}$ in order to produce a high current density of $10 \mathrm{~mA} / \mathrm{cm}^{2}$. It is notable that this experiment was preceded by a five time cycling between $-0.4 \mathrm{~V}$ and $0.0 \mathrm{~V}$ (vs. RHE) at a scan rate of $2 \mathrm{mV} / \mathrm{s}$. Fig. $\mathrm{S} 7$ depicts a stability test of a nanotruss-coated carbon-paper electrode, revealing that over $12 \mathrm{~h}$ of constant-current operation at $10 \mathrm{~mA} / \mathrm{cm}^{2}$ the overpotential vs. RHE is essentially unchanged. A movie depicting the corresponding vibrant formation of $\mathrm{H}_{2}$ gas bubbles from a nanotruss electrode is available in the Supporting Information (and Fig. S8). We have also calculated the exchange current density as a function of deposition time using the LSV data, and the observed linear relationship for deposition times $\leq 10$ min (data presented in Table S1) implies that also the longer nanotrusses remain well-connected with the carbon-paper substrate. Samples coated for longer time than 10 minutes showed an uneven growth with areas of very fluffy and low-density nanostructures, which had a higher tendency to peel off from the substrate. Therefore, it is not possible to achieve reliable tests of the catalytic performance for these samples. 

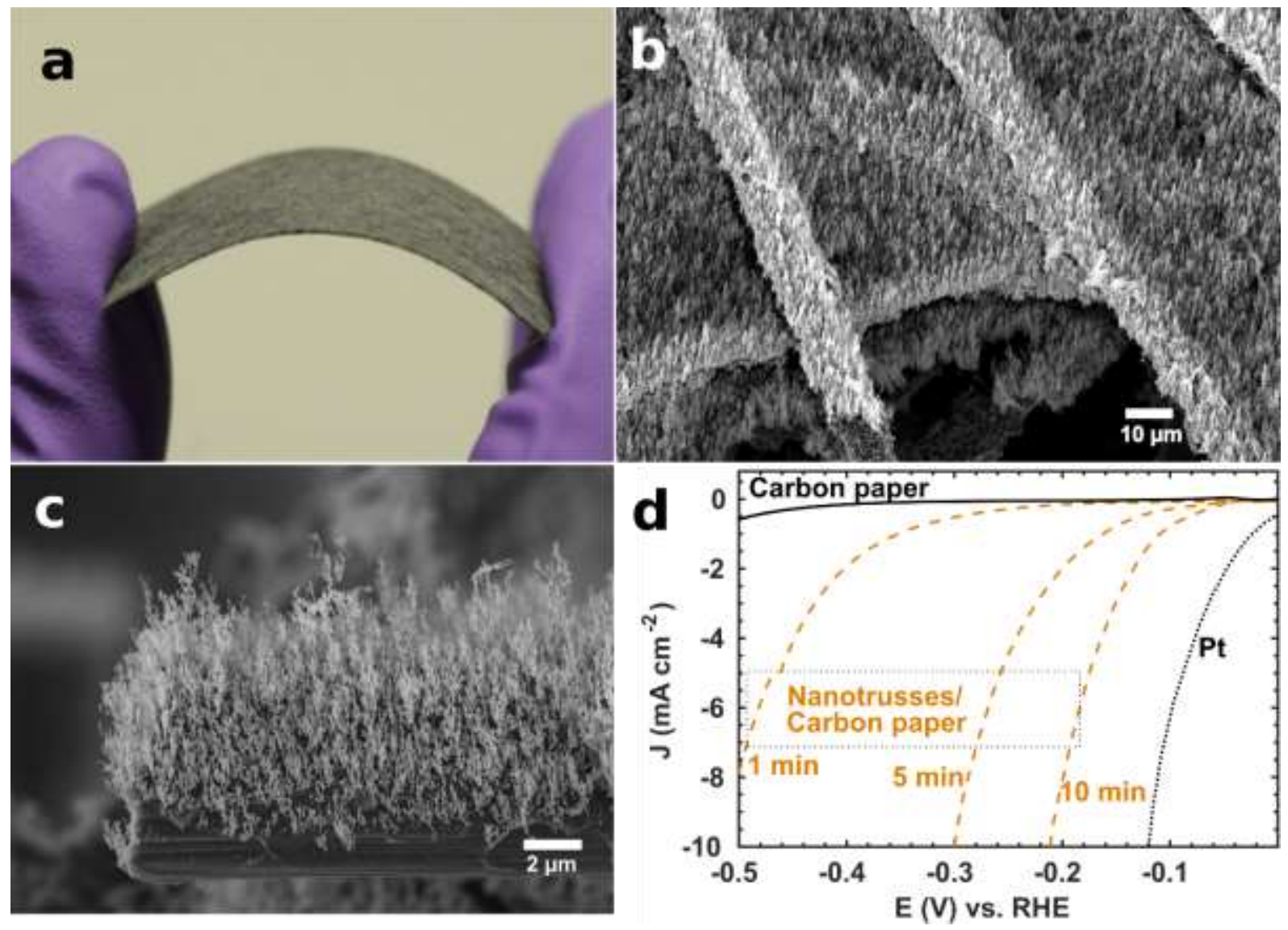

Figure 3. Nanotrusses as catalysis electrode material (a) Photograph of the bent carbon-paper substrate. (b) Low-magnification SEM image of a nanotruss coated carbon-paper substrate. The long (bright) carbon fibers with a typical diameter of $10 \mu \mathrm{m}$ feature a wire-network morphology, with each carbon fiber being coated with a film of nanotrusses. (c) Side-view SEM highlighting the dense mat of nanotrusses on top of a carbon fiber. (d) Linear sweep voltammetry of a bare carbon paper (solid line), carbon paper coated by nanotrusses (dashed lines) with the coating time marked in the graph, and a Pt wire (dotted line). The counter electrode was a Pt wire, and the electrolyte solution was $0.1 \mathrm{M} \mathrm{KOH}(\mathrm{pH}=13)$.

Importantly, to our knowledge, the herein reported data for hydrogen splitting from water for the 10-min-nanotrusses-on-carbon-paper catalyst-electrode compares favourably with previous studies on single transition-metal-oxides catalyst-electrodes synthesized by conventional chemical 
methods (see Table S2), and is in fact only $~ 90 \mathrm{mV}$ higher than that of the expensive standard in the field, platinum (see the dotted line in Fig. 3d). We finally wish to call attention to that this catalyst-electrode is both light-weight and flexible (see Fig. 3a), and as such can offer a very high capacity per both volume and mass, and that all constituent materials (Fe, $\mathrm{O}, \mathrm{C})$ are environmentally benign, low cost and plentiful.

In summary, we have shown that networked nanostructures, nanotrusses, can be directly selfassembled from Fe nanoparticles generated in a low-pressure pulsed plasma. The nanoparticles do not assemble in the plasma due to a barrier raised by the charge of the nanoparticles. However, at the substrate this barrier is removed and all nanostructures in contact with the substrate are uncharged. Therefore, approaching nanoparticles will self-assemble due to magnetic interactions. This results in nanowires growing out from the substrate. If a polydisperse size distribution of nanoparticles is used, magnetic leak-fields in the nanowires will create preferential sites for branching and linking. Hence, by controlling the size distribution of nanoparticles, we can tailor the branching of the nanostructures and shape these, into either single nanowires or nanotrusses, on demand. The use of controlled plasma environment allows for pure Fe nanoparticles to be generated, and as a consequence the assembled nanotrusses consist of a highly electrically conducting core while the surface is oxidized in the exposure to air after the process. The nanotrusses obtain a huge surface that, if grown on a conducting surface, have potential to generate highly performing electrodes. We have demonstrated this electrode performance in a water splitting experiment where hydrogen gas could be generated with a low overpotential.

\section{Methods}

The nanoparticles where synthesized using hollow cathode sputtering in a high vacuum system with a base pressure of approximately $3 \times 10^{-7}$ Torr $\left(4 \times 10^{-5} \mathrm{~Pa}\right)$. The arrangement is schematically 
illustrated in Fig. 1a. The cathode, with an inner diameter of $5 \mathrm{~mm}$ and a length of $54 \mathrm{~mm}$, consisted of Fe with a purity of $99.95 \%$. The cathode was mounted in a copper block, which in turn was placed on a magnetron (with its magnets removed) to provide electrical connection and water cooling. The copper block was isolated from the plasma using Kapton tape. A cylindrical mesh, with a diameter of $85 \mathrm{~mm}$ and a length of $110 \mathrm{~mm}$ was placed in front of the cathode to confine the plasma. The mesh was kept at floating potential throughout the experiments. Around $20 \mathrm{~mm}$ from the exit of the cathode, a grounded anode was placed. The anode, made from stainless steel, was in the form of a ring with a diameter of $35 \mathrm{~mm}$. The support rods that holds the actual ring in place was covered with Kapton tape to make sure that only the ring acted as anode. As sputter gas, Ar with a purity of $99.9997 \%$, was let in through the hollow cathode with a flow rate of $70 \mathrm{sccm}$. To ensure nucleation of nanoparticles, $\mathrm{O}_{2}$ was let in to the chamber about $30 \mathrm{~cm}$ downstream from the cathode exit. The $\mathrm{O}_{2}$ was diluted with Ar resulting in an effective $\mathrm{O}_{2}$ flow of $0.025 \mathrm{sccm}$. A throttle valve was used to keep the working pressure around 0.8 Torr (105 Pa) during experiments. The electric power to the cathode was supplied using a pulsed power supply (HiPSTER 1, Ionatics AB, Sweden). The pulsing frequency was kept at $1200 \mathrm{~Hz}$ and the pulse width at $80 \mu$ s throughout the experiments. The average output power was kept constant, at $75 \mathrm{~W}$. A permanent magnet and substrate were placed as shown in fig. 1a. The magnet had a diameter of $9 \mathrm{~mm}$, a height of $7 \mathrm{~mm}$ and a magnetic flux density, at the center of the surface facing the plasma, of $0.36 \mathrm{~T}$. Substrates were attached to the magnets by the use of double-sided copper tape and all substrates were quadratic with $10 \mathrm{~mm}$ side. The Si wafer substrates were coated with a $200 \mathrm{~nm}$ thick Ti film to make them conductive. The carbon paper, Sigracet GDL 34 AA, was purchased from Ion Power, Nafion ${ }^{\mathrm{TM}}$ Store, US. The substrates were in all experiments kept at floating potential. No shutter was used between the cathode and substrate during the process, which means that the substrate was 
exposed to nanoparticle "collection" from the moment the power supply was turned on. When NPs were collected without a permanent magnet, as in Supporting Information Fig S3, the nanowires assembled at one of the $90^{\circ}$ angles on the anode seen in Fig. 1a. All SEM images, except the ones on carbon paper, were taken using a LEO 1550 Gemini. The images with carbon paper were taken with a Zeiss Merlin FEG-SEM microscope. The TEM images were taken using a FEI Tecnai G2. For TEM samples, substrates with the nanostructures were ultra-sonicated in isopropanol and TEM grids with an amorphous carbon support layer were then dipped in the solution and left to dry. Comsol Multiphysics version 5.3 has been used for the numeric magnetic and electric field calculations. The catalytic capacity of the Nanotruss/Carbon Paper electrode was investigated by linear sweep voltammetry using a three-electrode cell connected to a potentiostat (MetrohmAutolab-PGSTAT302N). The working electrodes were prepared by cutting $0.2 \mathrm{~cm}^{2}$ pieces of bare or nanotruss-coated carbon paper. The reference electrode was $\mathrm{Ag} / \mathrm{AgCl}$ (CH1111-CH instruments), the counter electrode was a Pt coil (99.999\%), and the electrolyte solution was $0.1 \mathrm{M}$ $\mathrm{KOH}(\mathrm{pH}=13)$. The scan rate was $0.2 \mathrm{mV} / \mathrm{s}$ and the potentials vs. $\mathrm{Ag} / \mathrm{AgCl}$ were transformed to the reversible hydrogen electrode (RHE) scale with the following equation: V (vs. RHE) = V (vs. $\mathrm{Ag} / \mathrm{AgCl})+0.222+0.059 \times \mathrm{pH}$. The potentials were compensated ex situ for the $\mathrm{iR}$ drop between the working and reference electrodes.

Supporting information Available:

SEM analysis of nanotrusses, (S)TEM analysis describing the nature of the oxide layer that surrounds the Fe nanoparticles and the assembled nanotrusses, SEM image of nanowires grown without external magnet, TEM image of interparticle interface between two nanoparticles collected without an external magnet, TEM images of nanotrusses and nanowires after being dispersed in isopropyl alcohol and ultrasonicated, analytic model for the energy barrier against agglomeration, 
nanoparticle capture zone estimations, interaction between two magnetized nanoparticles in a plasma calculations, electrocatalysis: description and measurements

\section{Acknowledgement}

The authors would like to thank the Knut and Alice Wallenberg Foundation for generous financial support and Ionautics $\mathrm{AB}$ for the use of pulsed power supplies and related technical support given by Petter Larsson.

\section{References}

1. Nedyalkov, N., Nakajima, N. \& Terakawa, M. Appl. Phys. Lett. 2016, 108, 043107.

2. Kou, Z., Liu, E., Yue, J., Sui, Y., Huang, Z., Zhang, D., Wang, Y., Zhai, Y., Du, J. \& Zhai, H. J. Appl. Phys. 2015, 117, 17E709

3. Donaldson, J.G., Linse, P. \& Kantorovich, S.S. Nanoscale 2017, 9, 6448-6462.

4. Zhang, X., Li, Y., Liu, R., Rao, Y., Rong, H. \& Qin, G. ACS Appl. Mater. Interfaces 2016, 8, 3494-3498

5. Wang, L., Lu, X., Wang, J., Yang, S. \& So, X. J. Alloys Compd 2016, 681, 50-56

6. Liu, J., Cao, M.-S., Luo, Q., Shi, H.-L., Wang, W.-Z. \& Yuan, J. ACS Appl. Mater. Interfaces 2016, 8, 22615-22622

7. Krajewski, M., Brzozka, K., Lin, W. S., Lin, H. M., Tokarczyk, M., Borysiuk, J., Kowalski, G. \& Wasik, D. Phys. Chem. Chem. Phys. 2016, 18, 3900-3909 
8. Kralj, S. \& Makovec, D. ACS Nano 2015, 9, 9700-9707

9. Bharti, B., Fameau, A.-L., Rubinstein, M. \& Velev, O.D. Nat. Mater. 2015, 14, 1104-1110

10. Yin, J., Li, X., Lv, F., Fan, Q., Zhao, Y.-Q., Zhang, Q., Wang, W., Cheng, F., Xi, P. \& Guo, S. ACS Nano 2017, 11, 2275-2283

11. Choudhary, N., Li, C., Chung, H.-S., Moore, J., Thomas, J. \& Jung, Y. ACS Nano 2016, 10, 10726-10735

12. Zhang, W., Qi, J., Liu, K. \& Cao, R. A. Adv. Energy Mater. 2016, 6, 1502489

13. Shimizu, T., Aoki, K., Tanaka, Y., Terui, T. \& Shingubara, S. Jpn. J. Appl. Phys. 2011, 50, 06GE01

14. Fang, X., Cheng, X., Zhang, Y., Zhang, L. G., Keidar, M. J. Colloid Interface Sci. 2018, 509, $414-421$

15. Bouchole, A. Dusty Plasmas: Physics, Chemistry and Technological Impacts in Plasma Processing. John Wiley Sons Ltd 1999

16. Polonski, O., Peter, T., Ahadi, A.M., Hinz, A., Strunskus, T., Zaporojtchenko, V., Biederman, H. \& Faupel, F. Appl. Phys. Lett. 2013, 103, 033118

17. Pilch, I., Söderström, D., Hasan, M.I., Helmersson, U. \& Brenning, N. Appl. Phys. Lett. 2013, 103, 193108

18. Ahadi, A. M., Zaporojtchenko, V., Peter, T., Polonskyi, O., Strunskus, T., Faupel, F., J. Nanopart. Res. 2013, 15, 2125

19. Mangolini, L., Kortshagen, U., Phys. Rev. E 2009, 79, 026405 
20. Guo, W., Zeng, Z., Zhang, X., Peng, P., Tang, S., J Nanomater 2015, 897142

21. Tavakkoli, M., Kallio, T., Reynaud, O., Nasibulin, A. G., Johans, C., Sainio, J., Jiang, H., Kauppinen, E. I. \& Laasonen, K. Angew. Chem. 2015, 127, 4618-4621 


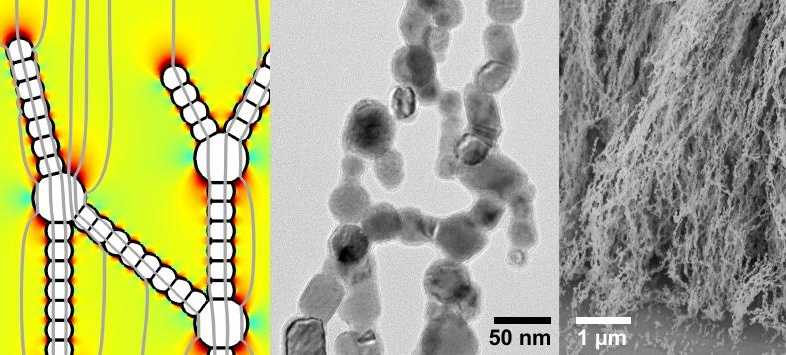



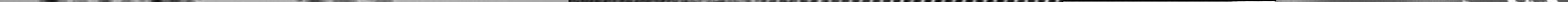


\section{Supporting Information}

\section{Catalytic nanotruss structures realized by magnetic}

\section{self-assembly in pulsed plasma}

Sebastian Ekeroth ${ }^{1}$, E. Peter Münger ${ }^{1}$, Robert Boyd ${ }^{1}$, Joakim Ekspong ${ }^{2}$, Thomas Wågberg ${ }^{2}$, Ludvig Edman ${ }^{2}$, Nils Brenning ${ }^{1,3}$, Ulf Helmersson ${ }^{1 *}$

1) Linköping University, Department of Physics, SE-581 83 Linköping, Sweden.

2) Umeå University, Department of Physics, SE-901 87 Umeå, Sweden.

3) KTH Royal Institute of Technology, School of Electrical Engineering, Division of Space and Plasma Physics, SE-100 44 Stockholm, Sweden.

*Corresponding author: ulf.helmersson@liu.se 


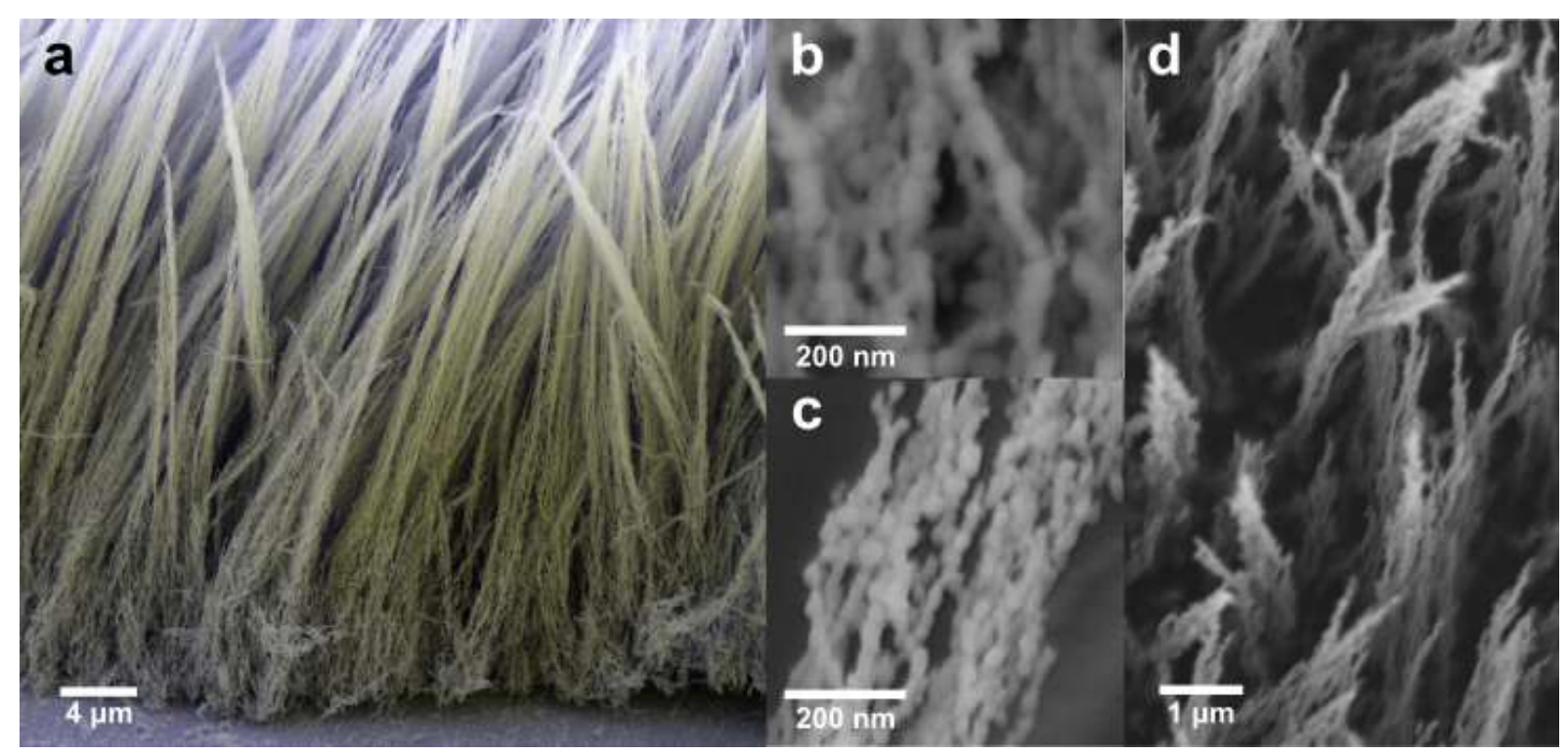

Figure S1. Scanning electron microscopy analysis of the nanotrusses. (a) a cross section image showing their entire structure. These structures also reveal the formation mechanism, as the particles adjacent to the substrate must have been deposited first. It can be seen that initially (close to the substrate) individual nanowires are formed that propagate upwards with relatively large distances between them (typically 50-150 nm) indicating little interaction, (b). With increasing nanotruss height the distance between individual wires reduces dramatically, often to within $20 \mathrm{~nm}$, (c). A top view image (d) shows that the nanotrusses terminate with the individual wires combining to form a single strand or point. 


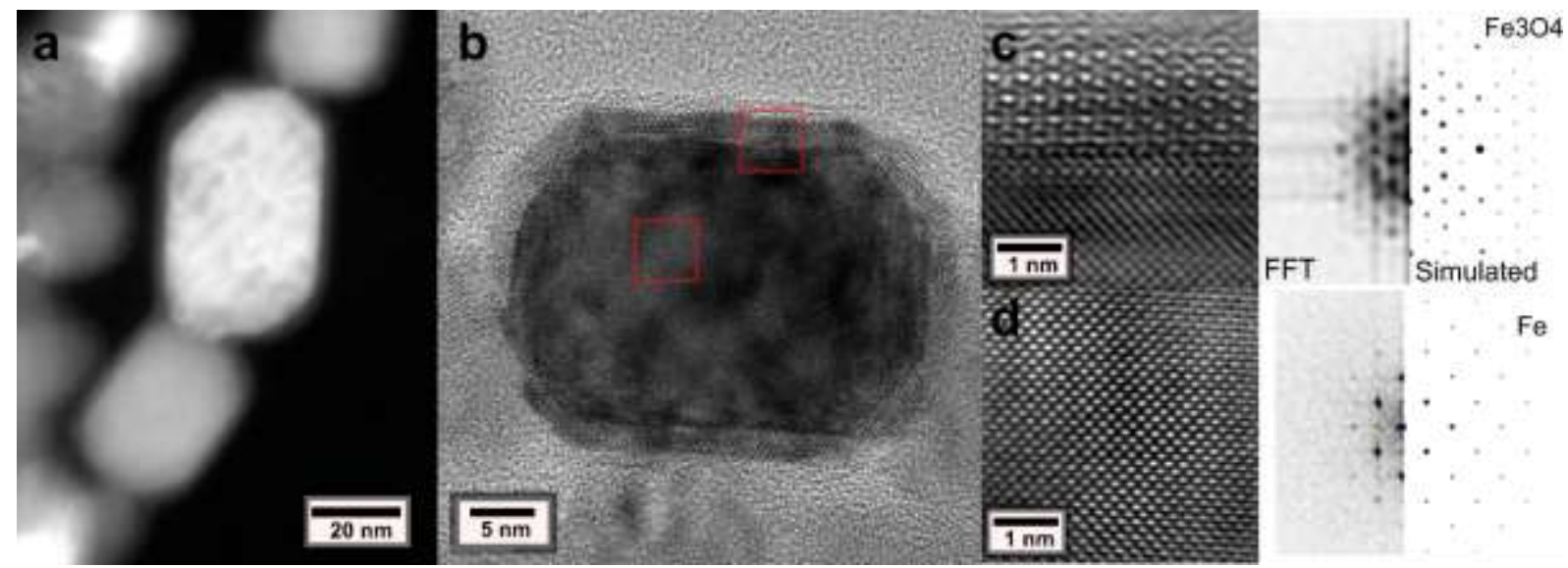

Figure S2. (S)TEM analysis describing the nature of the oxide layer that surrounds the Fe nanoparticles and the assembled nanotrusses. (a) HAADF-STEM image of, predominantly, three attached particles which form part of a longer wire. The core of the particles is clearly visible with a $2 \mathrm{~nm}$ thick 'halo' surrounding them, which corresponds to the oxide layer. Both types of particle attachments are highlighted. The middle and lower particles appear sintered together, whilst the middle and top particles appear attached by physical contact. Although in both cases the apparent area of interaction is smaller than the actual one due to influence of the oxide layer and is strong enough to withstand ultrasonic treatment. The presence of a metal core and oxide shell is confirmed by high resolution TEM analysis (b-d). (b) shows an overview of a single nanoparticle that forms part of a longer wire. The core shell structure is clear. The crystal structure of both the shell and core are apparent in high resolution images of the respective areas (c and $\mathrm{d}$ from the shell and core respectively). FFT patterns obtain from these images could be indexed to simulated patterns obtained from the $\mathrm{Fe}_{3} \mathrm{O}_{4}$ (zone axis $(1,1,0)$ ) and Fe (zone axis $(1,0,0))$ crystal structures for the shell and core respectively. Similar phenomena have been observed in previous studies. ${ }^{1,2}$ 


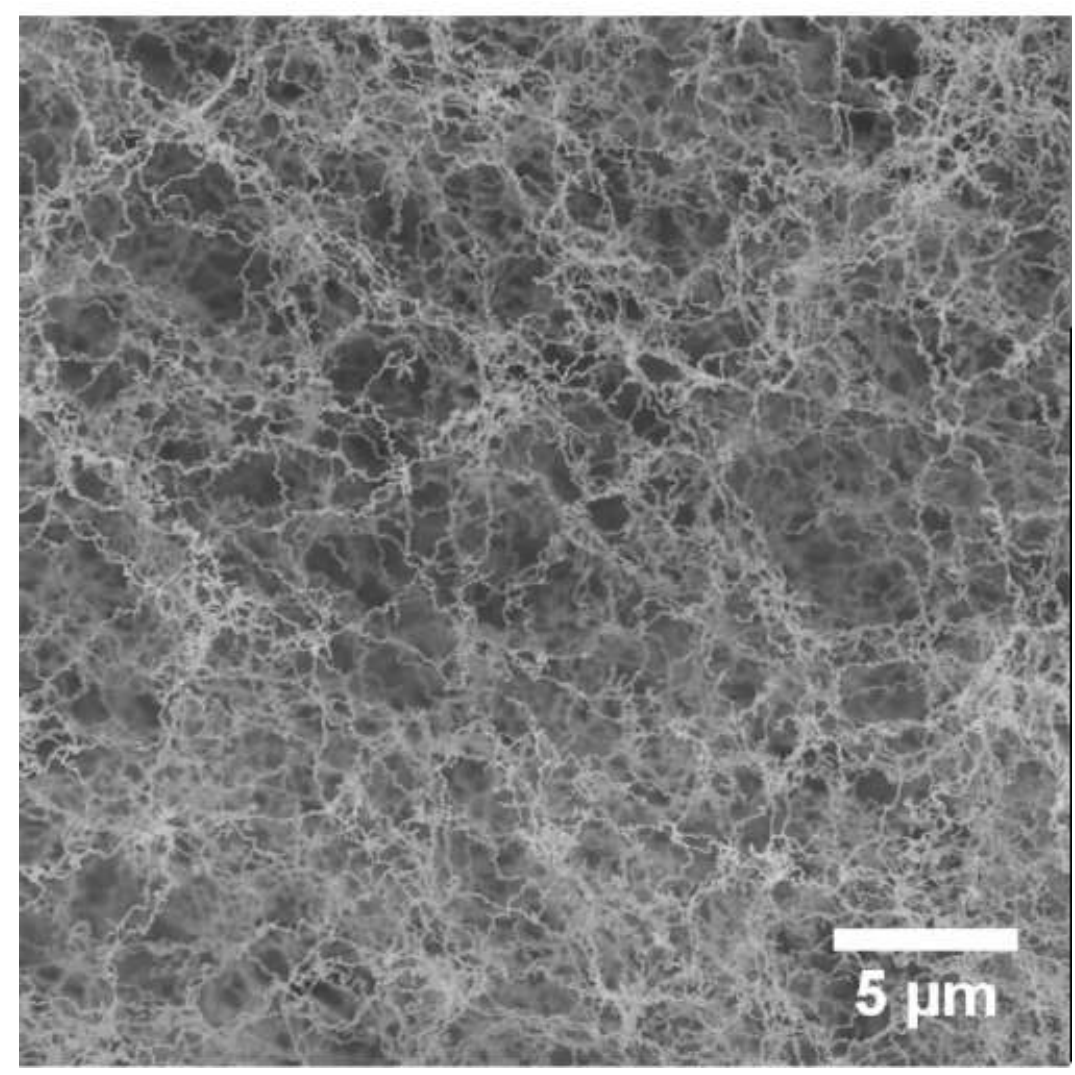

Figure S3. SEM image of nanowires grown without external magnet. Featuring nanowires of random orientation. 


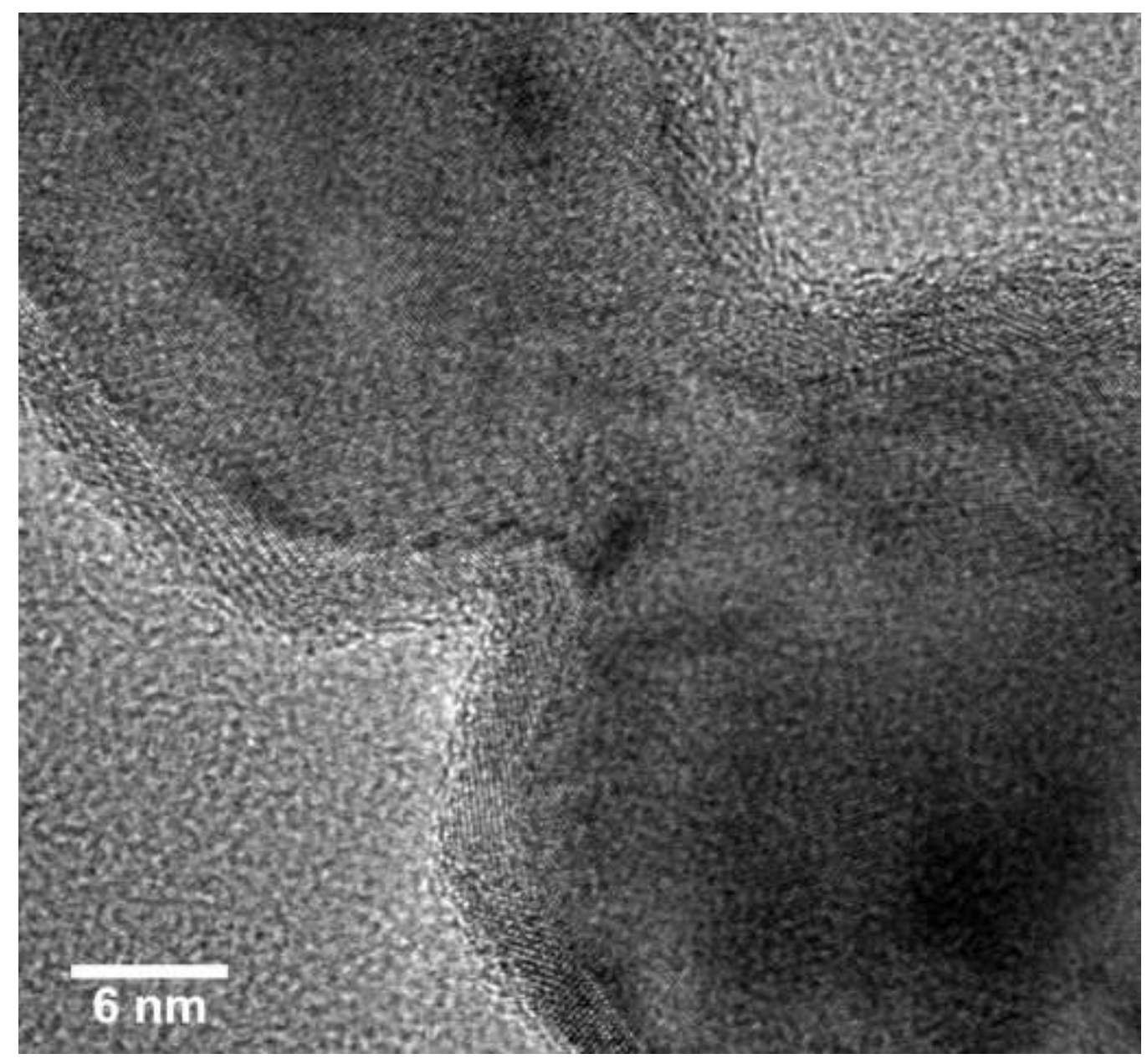

Figure S4. TEM image of interparticle interface between two nanoparticles collected without an external magnet. 


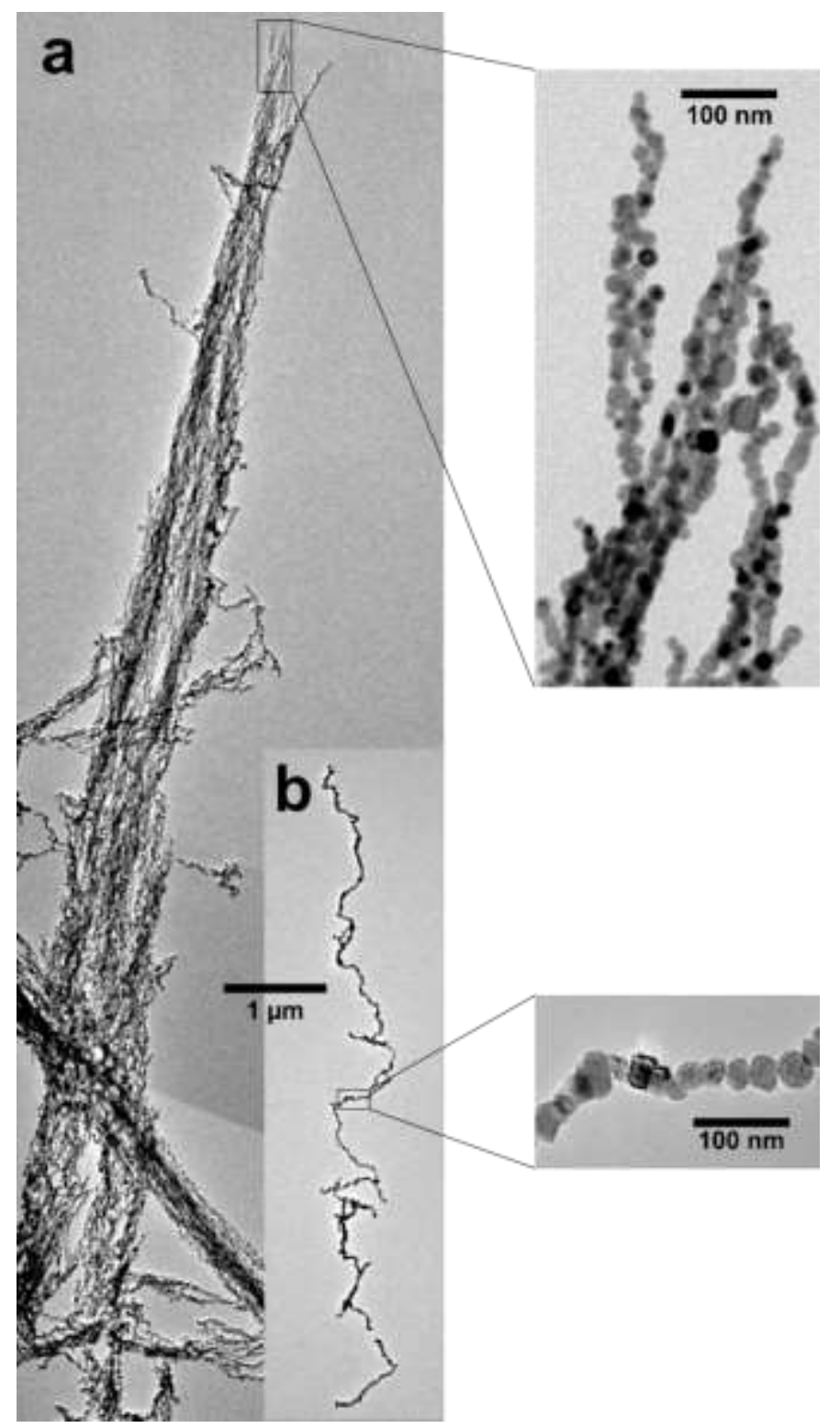

Figure S5. TEM images of nanotrusses and nanowires after being dispersed in isopropyl alcohol and ultrasonicated. (a) from nanotruss structures grown on a magnet, similar to sample in fig. 1b. (b) from sample grown without external magnet, similar to sample in fig S3.

The above figure highlights the microscopic differences in the structures consisting of iron nanoparticles produced with (a) and without (b) an applied magnetic field. Without the applied field long, sparsely branched wires are produced which can be easily separated by ultrasonic treatment. With the applied field, abundantly branched and cross-linked wires are produced which cannot be separated under ultrasonic treatment. 


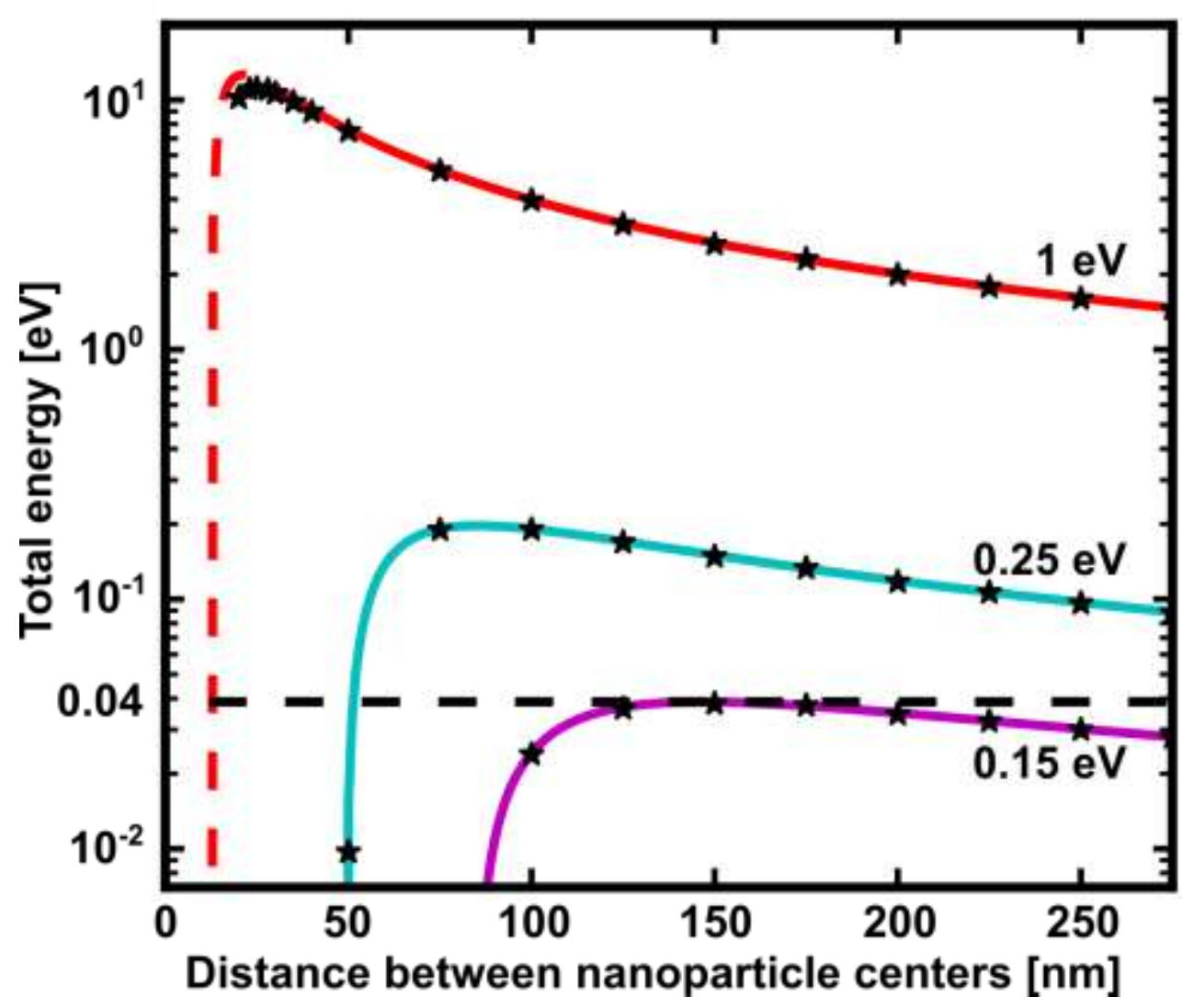

Figure S6. The energy barrier against agglomeration for nanoparticles with $20 \mathrm{~nm}$ diameter in a plasma with different electron temperatures, $1,0.25$ and $0.15 \mathrm{eV}$. The analytic model shows excellent agreement with Finite-Element-Method (FEM) calculations, black stars, for separations larger than 1.5 to 2 times the nanoparticle diameter. The dashed red line indicates separations where the analytic model is less good. The dashed black line indicates the average thermal translational energy for a nanoparticle in a gas at a temperature of $300 \mathrm{~K}(0.04 \mathrm{eV})$. 


\section{Section 1: Nanoparticle capture zone estimation.}

As a nanoparticle with the magnetic moment $\boldsymbol{m}$ is approaching an external magnet with the magnetic field $\boldsymbol{B}$ it will experience a magnetic potential energy of $\emptyset_{\mathrm{B}}=-\boldsymbol{m} \cdot \boldsymbol{B}$. Nanoparticles with a rotational energy lower than $|\boldsymbol{m} \boldsymbol{B}|$ will become more and more aligned with the external field as the field strength increases. The rotational energy can be estimated from the average thermal rotational energy $W_{\text {th,rot }}=\frac{3 k_{\mathrm{B}} T_{\mathrm{g}}}{2}$. Although the internal temperature of the NPs can be considerably higher than the process gas temperature $T_{\mathrm{g}}{ }^{3}$ their translation and rotation degrees of freedom are in equilibrium with the gas. Combined this give a critical field strength for alignment

$$
B_{C}=\frac{3}{2} k_{B} T_{g}\left(M \frac{4 \pi r_{N P}^{3}}{3}\right)^{-1}=\frac{9}{8} \frac{k_{B} T_{g}}{\pi r_{N P}^{3} M},
$$

where $M$ is the magnetization and $r_{N P}$ is the radius of the nanoparticle. When $B>B_{\mathrm{c}}$, the rotation of a nanoparticle becomes constrained to limited deviations from alignment between $\boldsymbol{m}$ and $\boldsymbol{B}$. Furthermore, nanoparticles are drawn towards regions with strong magnetic field in order to lower their average magnetic energy. For the special case where $\boldsymbol{m}$ and $\boldsymbol{B}$ are fully aligned, the magnetic potential energy is $-m B$. Nanoparticles are thus not only aligned but also trapped and cannot escape the magnetic potential well from an external magnet if their translational kinetic energy is lower than $m B$ for the fully aligned case*. We define a critical field strength for entrapment as when the depth of this well, $m B$, equals the average thermal kinetic energy $W_{\text {th,kin }}$ in translational motion of the nanoparticles. At thermal equilibrium $W_{\text {th,kin }}=W_{\text {th,rot }}$ and it follows that the critical field strength for magnetic capture is the same as $B_{\mathrm{c}}$ in Eq. $\mathrm{S} 1$ for alignment. Therefore, the critical field $B_{c}$ also define limits of the "capture

\footnotetext{
* Neglecting long-range electric forces, their initial speed as they form inside a gas flow, and the frictional drag between a moving nanoparticle and the neutral gas
} 
zone". The capture zone limit depends on the external magnetic field as well as on the magnetic properties and size of the nanoparticle. The capture zones, shown in Fig. $2 b$ are for spherical nanoparticles with different diameters, are calculated for the magnet used at the substrate position in our experiments. 


\section{Section 2: Interaction between two magnetized nanoparticles in a plasma.}

Nanoparticles in a plasma will be charged negatively creating a repulsive force. The interaction between two magnetized nanoparticles in a plasma will then be subjected to two competing forces - electrostatic and magnetostatic. The magnetic attraction is shorter ranged than the electrostatic repulsion and will dominate at small separation. Magnetized nanoparticles are thus repelled at large separation and attracted at close distances.

A simple model system is used to examine the interaction. Each nanoparticle is approximated as a point charge $q_{i}$ and magnetic dipole moment $\boldsymbol{m}_{i}$ with $i=1,2$ for the two nanoparticles. This approximation introduces errors due to form factors (the synthesized nanoparticles are not spherical), and due to induced mirror charges on the nanoparticle surfaces. Both kind of errors increases with decreasing separation between the nanoparticles. Comparison with numerical finite element calculations, as implemented in Comsol Multiphysics, for two spherical nanoparticles with $20 \mathrm{~nm}$ diameter shows excellent agreement as soon as the separation is large enough to fit at least an equally sized nanoparticle in between (Fig. 2a). This is a general conclusion due to geometric scaling. Using the electrostatic potential from a point charge and the magnetic field at large distance from a magnetic dipole moment the total electrostatic and magnetostatic energy for the two nanoparticles is

$$
W_{\mathrm{tot}}=W_{\mathrm{e}}+W_{m}=\frac{q_{1} q_{2}}{4 \pi \varepsilon_{0} z}-\frac{\mu_{0} m_{1} m_{2}}{2 \pi z^{3}}
$$

where $\varepsilon_{0}$ and $\mu_{0}$ are the vacuum permittivity and vacuum permeability, $m_{1}$ and $m_{2}$ are the magnitude of the two magnetic dipole moments and $z$ is the separation between the nanoparticles. $W_{\text {tot }}$ have a maximum, $W_{\max }$, at the separation $z_{\max }$ and is given by:

$$
W_{\max }=\frac{1}{\pi \sqrt{\mu_{0} m_{1} m_{2}}}\left(\frac{q_{1} q_{2}}{6 \varepsilon_{0}}\right)^{3 / 2}
$$




$$
z_{\max }=\sqrt{6 \varepsilon_{0} \mu_{0}} \sqrt{\frac{m_{1} m_{2}}{q_{1} q_{2}}}
$$

Hence, the nanoparticles have a barrier $W_{\max }$ to overcome in order to form a pair and they are repelled for separations larger than $z_{\max }$ and attracted for separations smaller than $z_{\max }$.

Further assumptions in the simple model is that the charge on the nanoparticles are independent of their separation and corresponds to a charge $q_{i}=4 \pi \varepsilon_{0} r_{i} V_{i}$ on a single sphere with radius $r_{i}$ at a potential $V_{i}$. With a homogeneous magnetization $M_{i}$ in the corresponding sphere the magnetic moment is $m_{i}=\frac{4 \pi r_{i}^{3}}{3} M_{i}$. Cast in these variables equation (S3a) and (S3b) becomes

$$
\begin{aligned}
& W_{\text {max }}=8 \pi \frac{\left(\varepsilon_{0} V_{1} V_{2}\right)^{3 / 2}}{\sqrt{6 \mu_{0} M_{1} M_{2}}} \\
& Z_{\text {max }}=\sqrt{\frac{2 \mu_{0} M_{1} M_{2}}{3 \varepsilon_{0} V_{1} V_{2}}} r_{1} r_{2}
\end{aligned}
$$

In a plasma, particles normally acquire the so-called floating potential, at which the net current to a particle is zero** ${ }^{*}$ By way of example, for an Argon plasma used in accordance with the present setup, the floating potential is $V_{\text {float }} \approx-2.41 k_{B} T_{\mathrm{e}} / e$, where $T_{\mathrm{e}}$ is the electron temperature, $k_{B}$ the Boltzmann constant and $e$ the elementary charge. ${ }^{4}$ With this potential and the same magnetization on both nanoparticles, $V_{1}=V_{2}=V_{\text {float }}$ and $M_{1}=M_{2}=M$, the barrier against agglomeration of nanoparticles in the volume is

$$
W_{\max }=\frac{8 \pi}{M} \sqrt{\frac{\varepsilon_{0}^{3}}{6 \mu_{0}}}\left(\frac{2.41 k_{B} T_{\mathrm{e}}}{e}\right)^{3}
$$

\footnotetext{
${ }^{* *}$ In a pulsed high density plasma, such charging occurs for all nanoparticles in the size range of above $10 \mathrm{~nm}$. For smaller nanoparticles, electron field emission can sometimes reduce the negative charge (the size for this to happen depends on the plasma density and the electron temperature). However, also with reduced charge the nanoparticles can be estimated to have sufficient negative charge to avoid agglomeration down to a few nm size.
} 


$$
Z_{\max }=\sqrt{\frac{2 \mu_{0}}{3 \varepsilon_{0}}} \frac{M e}{2.41 k_{B} T_{e}} r_{1} r_{2}
$$

Note that the height of the barrier decreases with decreased electron temperature (Fig. S6), which gives less charge on the nanoparticles, and at the same time the separation at the maximum increases. The height of the barrier is to be compared to the typical thermal kinetic energy for translational motion $W_{\text {th,kin }}=\frac{3 k_{B} T_{\mathrm{g}}}{2}$ for nanoparticles in thermal equilibrium with the surrounding process gas at the temperature $T_{\mathrm{g}}$. At room temperature, $T_{\mathrm{g}}=300 \mathrm{~K}, W_{\mathrm{th} \text {,kin }} \approx$ $3.88 \cdot 10^{-2} \mathrm{eV}$. For agglomeration to be likely the thermal translational energy has to be large enough to overcome the barrier, $W_{\text {th,kin }}>W_{\text {max }}$, which is fulfilled if the magnetization is $M=$ $1.67 \cdot 10^{6} \mathrm{~A} / \mathrm{m}$ (the value for Fe) and $T_{\mathrm{e}}<0.15 \mathrm{eV}$. At higher $T_{\mathrm{e}}$, agglomeration is suppressed by strong electrostatic repulsion due to the charge on the nanoparticles. It should be noted that this condition overestimates the probability for agglomeration, since head-on collisions combined with magnetic alignment is an unlikely geometry in random collisions between nanoparticles that both rotate and have random velocities, even though magnetic interactions tend to align the magnetic dipole moments of nanoparticles.

For reference, it can be noted that the electron temperature in the nanoparticle growth zone of our type of plasma discharge has been estimated to be in the order of $1 \mathrm{eV} .^{5}$ The condition above for agglomeration is, surprisingly, found to be independent of the sizes of the two nanoparticles involved. If dimers cannot be formed in the gas/plasma phase, then the road to the formation of longer nanowires in the plasma is also closed. 


\section{Section 3: Catalysis}

In a water-splitting experiment under alkaline conditions, $\mathrm{H}_{2}$ and $\mathrm{O}_{2}$ gas are formed at the cathode and anode, respectively, in accordance with the following equations:

$\begin{array}{ll}\text { Cathode: } & 4 \mathrm{H}_{2} \mathrm{O}+4 \mathrm{e}^{-} \rightarrow 2 \mathrm{H}_{2}(\mathrm{~g})+4 \mathrm{OH}^{-} \\ \text {Anode: } & 4 \mathrm{OH}^{-} \rightarrow \mathrm{O}_{2}(\mathrm{~g})+2 \mathrm{H}_{2} \mathrm{O}+4 \mathrm{e}^{-} \\ \text {Overall: } & 2 \mathrm{H}_{2} \mathrm{O} \rightarrow 2 \mathrm{H}_{2}(\mathrm{~g})+\mathrm{O}_{2}(\mathrm{~g})\end{array}$

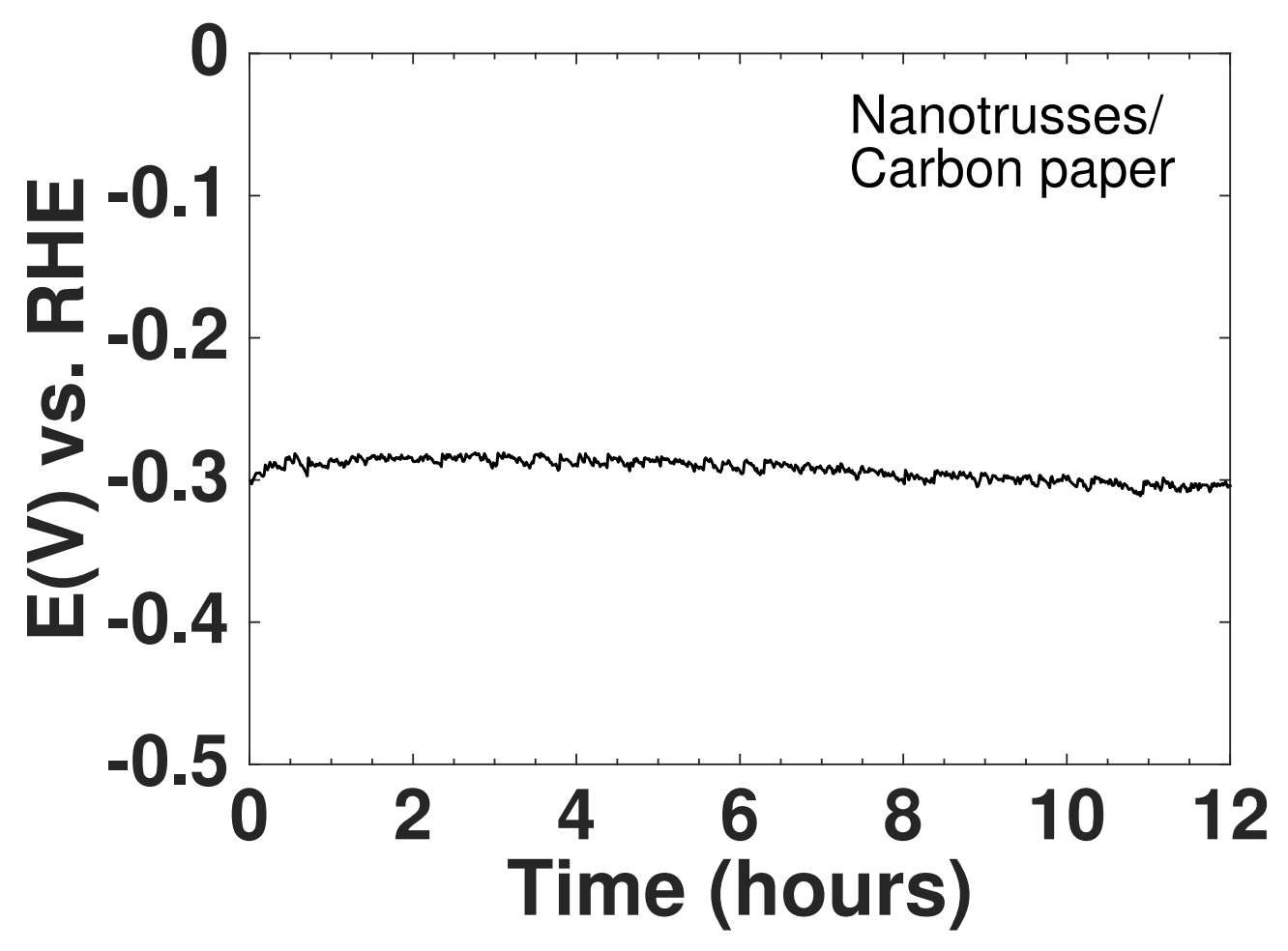

Figure S7. The long-term stability of a nanotruss coated carbon-paper electrode (overpotential of $0.3 \mathrm{~V}$ ) when driven at a constant current of $-10 \mathrm{~mA} \mathrm{~cm}^{-2}$ for 12 hours. The electrolyte solution comprised $0.1 \mathrm{M} \mathrm{KOH}$.

In line with the above, the photograph in Fig. $\mathrm{S} 8$ depicts a vibrant formation of $\mathrm{H}_{2}$ gas bubbles at the (black) nanostructured cathode to the left and $\mathrm{O}_{2}$ gas bubbles at the Pt-wire anode to the right. A movie of the process is included in the Supporting Information. 


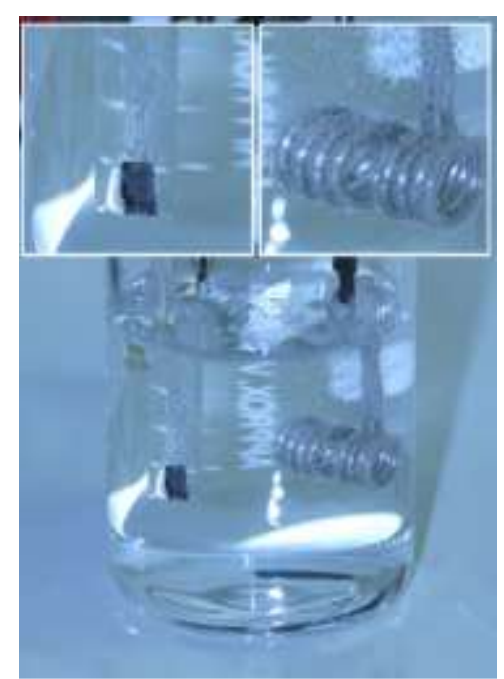

Figure S8. A photograph of the electrochemical reaction beaker in which $\mathrm{H}_{2}$ gas bubbles are formed at the (black) Nanotrusses/Carbon paper cathode to the left and $\mathrm{O}_{2}$ gas bubbles are released from the Pt-coil to the right. 


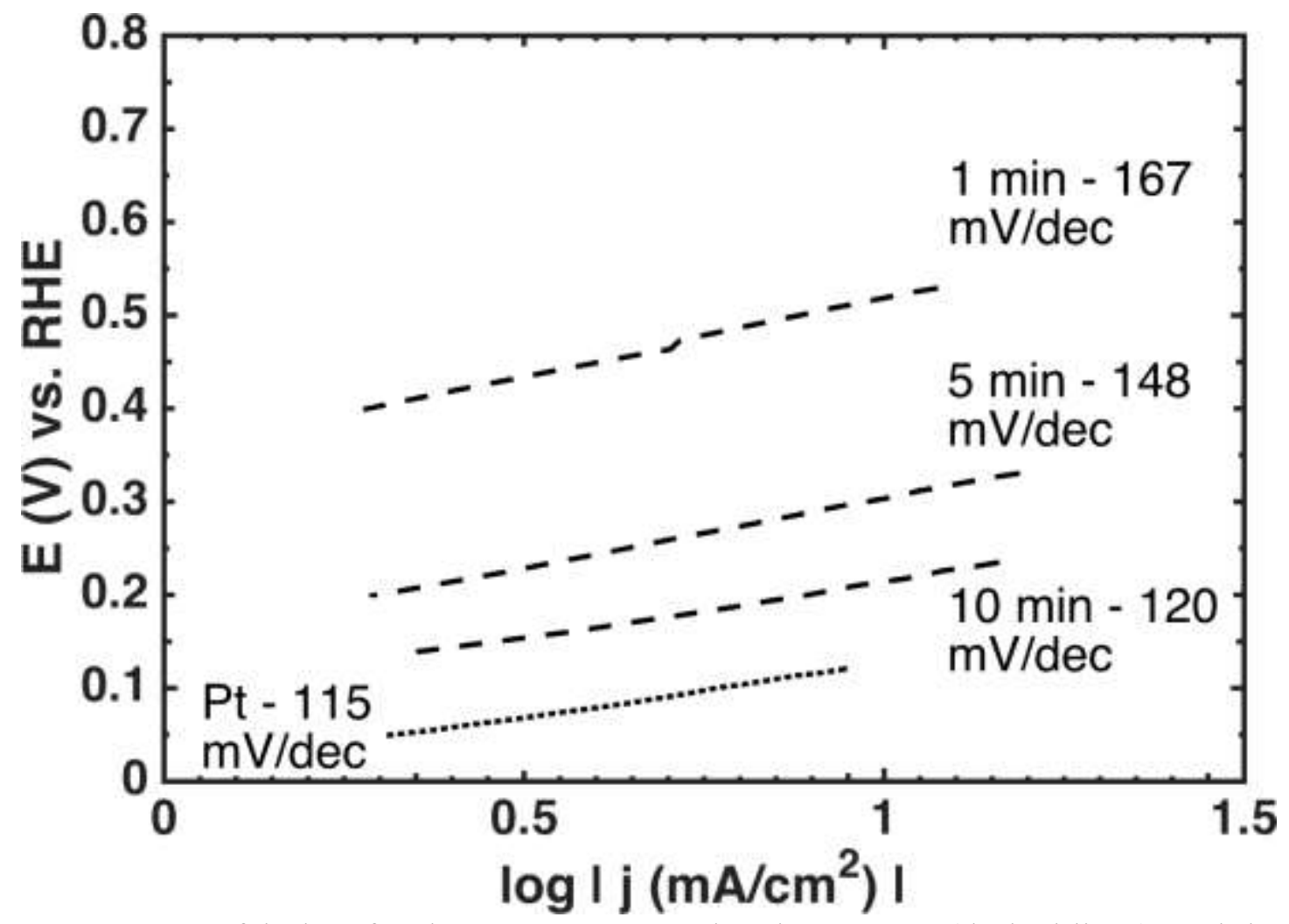

Figure S9. Tafel plots for the nanotruss-coated carbon papers (dashed lines), and the Pt wire (dotted line). The electrolyte was a $0.1 \mathrm{M} \mathrm{KOH}$ solution $(\mathrm{pH}=13)$ in all experiments and all data is derived from the LSV in Fig. $3 \mathrm{~d}$.

Table S1. The Tafel slope, the exchange current density, and the overpotential for the nanotruss-coated carbon papers and the Pt-wire. The values were derived from data presented in Fig. 3d and S9.

\begin{tabular}{|l|l|l|l|}
\hline Electrode & Tafel slope $(\mathrm{mV} / \mathrm{dec})$ & $i_{0}\left(\mu \mathrm{A} / \mathrm{cm}^{2}\right)$ & $\begin{array}{l}\text { Overpotential } \\
\left(@-10 \mathrm{~mA} / \mathrm{cm}^{2}\right)\end{array}$ \\
\hline $\begin{array}{l}\text { Nanotrusses/Carbon } \\
\text { paper 1 min }\end{array}$ & 167 & 8.00 & $520 \mathrm{mV}$ \\
\hline $\begin{array}{l}\text { Nanotrusses/Carbon } \\
\text { paper 5 min }\end{array}$ & 148 & 91.1 & $300 \mathrm{mV}$ \\
\hline $\begin{array}{l}\text { Nanotrusses/Carbon } \\
\text { paper 10 min }\end{array}$ & 120 & 167 & $210 \mathrm{mV}$ \\
\hline Pt-wire (reference) & 115 & 790 & $120 \mathrm{mV}$ \\
\hline
\end{tabular}


Table S2. Comparison between different metal catalysts for HER in alkaline electrolytes.

\begin{tabular}{|l|l|l|l|}
\hline Electrode & Electrolyte & $\begin{array}{l}\text { Overpotential } \\
\left(@-10 \mathrm{~mA} / \mathrm{cm}^{2}\right)\end{array}$ & Reference \\
\hline NiOH on Ni foam & $1.0 \mathrm{M} \mathrm{NaOH}$ & $250 \mathrm{mV}$ & 6 \\
\hline $\begin{array}{l}\mathrm{NiO} / \mathrm{Ni} \text { on carbon } \\
\text { nanotubes }\end{array}$ & $1.0 \mathrm{M} \mathrm{KOH}$ & $100 \mathrm{mV}$ & 7 \\
\hline $\begin{array}{l}\mathrm{Co}_{3} \mathrm{O}_{4} \text { nanoparticles } \\
\text { on carbon paper }\end{array}$ & $0.1 \mathrm{M} \mathrm{KOH}$ & $380 \mathrm{mV}$ & 8 \\
\hline $\begin{array}{l}\text { CoOx on N-doped } \\
\text { carbon }\end{array}$ & $1.0 \mathrm{M} \mathrm{KOH}$ & $235 \mathrm{mV}$ & 9 \\
\hline $\begin{array}{l}\text { Metallic } \\
\text { nanoparticulates } \\
\text { supported on FTO }\end{array}$ & $0.1 \mathrm{M} \mathrm{KOH}$ & $420 \mathrm{mV}$ & 10 \\
\hline $\begin{array}{l}\text { Nanotrusses/Carbon } \\
\text { paper }\end{array}$ & $0.1 \mathrm{M} \mathrm{KOH}$ & $210 \mathrm{mV}$ & This work \\
\hline
\end{tabular}


References

1. Pratt, A., Lari, L., Hovorka, O., Shah, A., Woffinden, C., Tear, S. P., Binns, C., Kröger, R., Nat. Mater. 2014, 13, 26-30

2. Lovely, G. R., Brown, A. P., Brydson, R., Kirkland, A. I., Meyer, R. R., Chang, L. Y., Jefferson, D. A., Falke, M., Bleloch, A., Micron, 2006, 37, 389-395

3. Kramer, N. J., Anthony, R. J., Mamunuru, M., Aydil, E. S. \& Kortshagen, U.R., J. Phys. D: Appl. Phys. 2014, 47, 075202

4. Pilch, I., Söderström, D., Hasan, M.I., Brenning, N. \& Helmersson, U., Appl. Phys. Lett. 2013, 103, 033108

5. Pilch, I., Söderström, D., Hasan, M.I., Helmersson, U. \& Brenning, N., Appl. Phys. Lett. 2013, 103, 193108

6. Luo, J., Im, J.H., Mayer, M.T., Schreier, M., Nazeeruddin, M.K., Park, N.G., Tilley, S.D., Fan, H.J. \& Grätzel, M., Science. 2014, 345, 1593-1596

7. Gong, M., Zhou, W., Tsai, M.-C., Zhou, J., Guan, M., Lin, M.-C., Zhang, B., Hu, Y., Wang, D.-Y., Yang, J., Pennycook, S.J., Hwang, B.-J. \& Dai, H., Nat. Commun. 2014, 5, 4695

8. Sharifi, T., Gracia-Espino, E., Jia, X., Sandström, R. \& Wågberg, T., Appl. Mat. Int. 2015, 7, 28148-28155

9. Haiyan, J., Jing, W., Diefeng, S., Zhongzhe, W., Zhenfeng, P. \& Yong. W., Jour. Am. Chem. Soc. 2015, 137, 2688-2694

10. Martindale, B. C. M. \& Reisner, E., Adv. Ener. Mat. 2016, 6, 1502095 


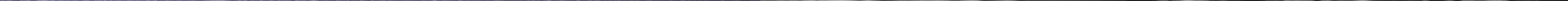




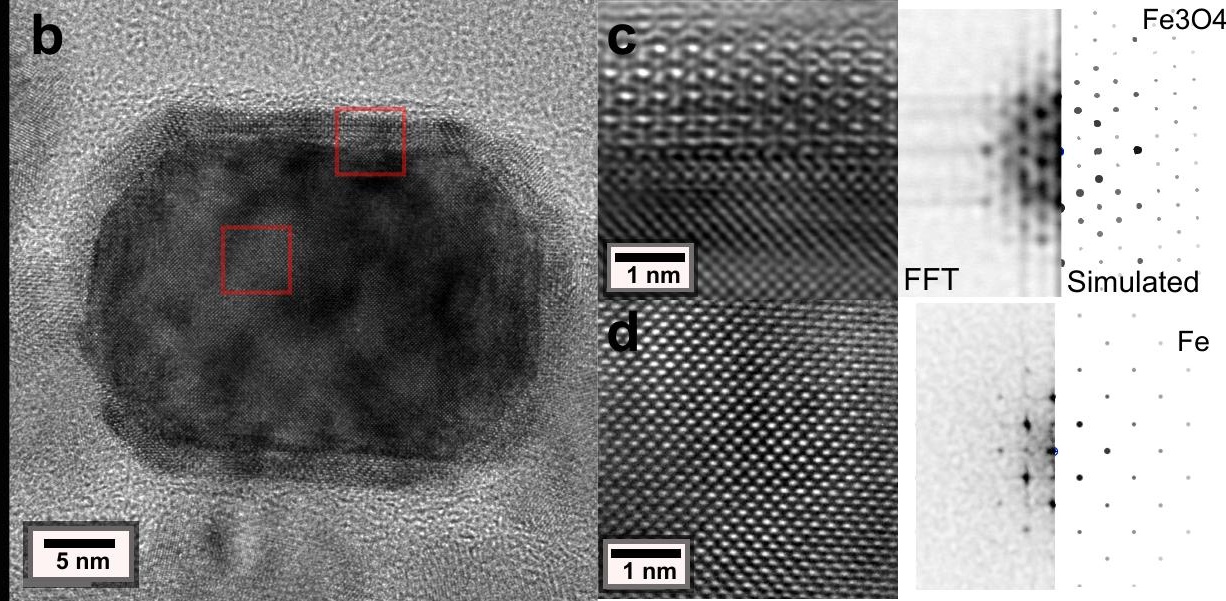




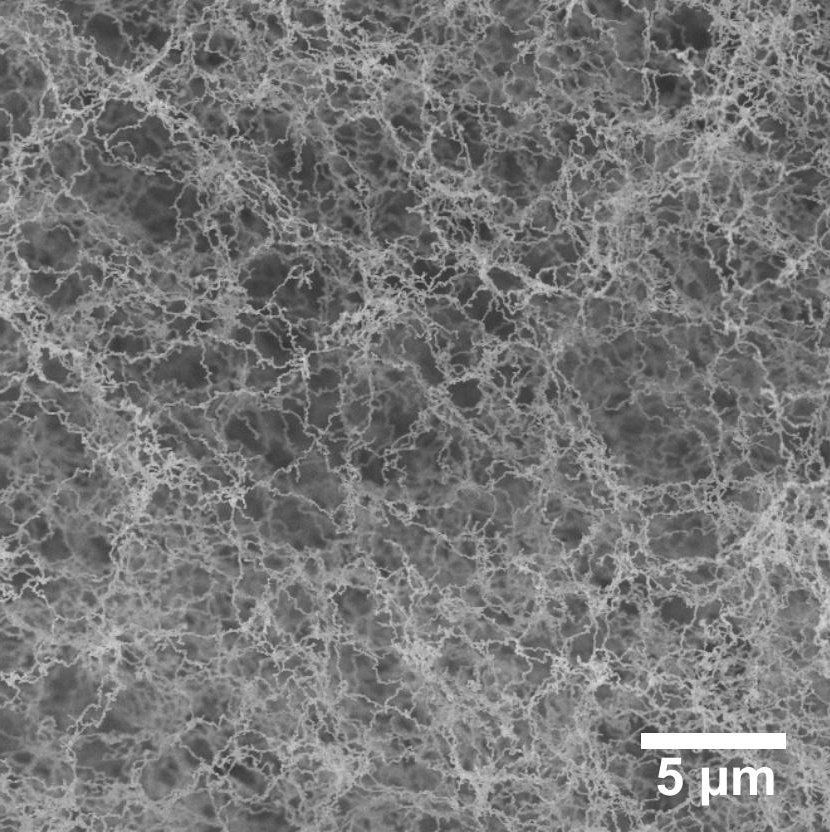


2. W. and

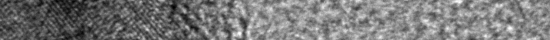

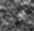

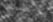
Cor.

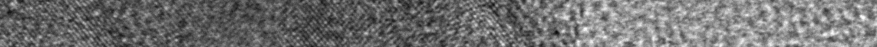

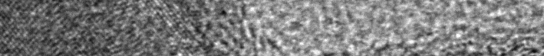

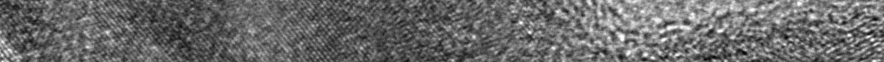

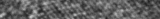

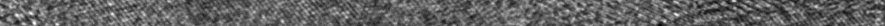

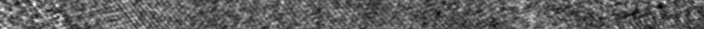

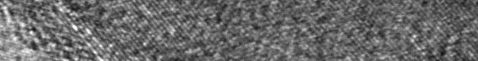

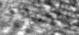

atsing W.

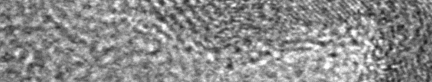

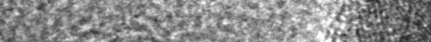

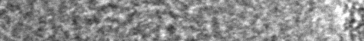

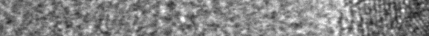

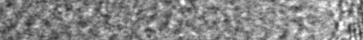

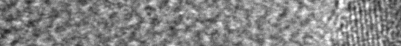

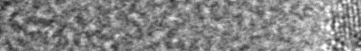

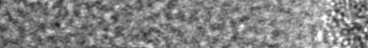

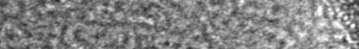

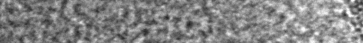

10.

$66 \mathrm{~nm}$

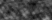

(x)

+4 . 10. 


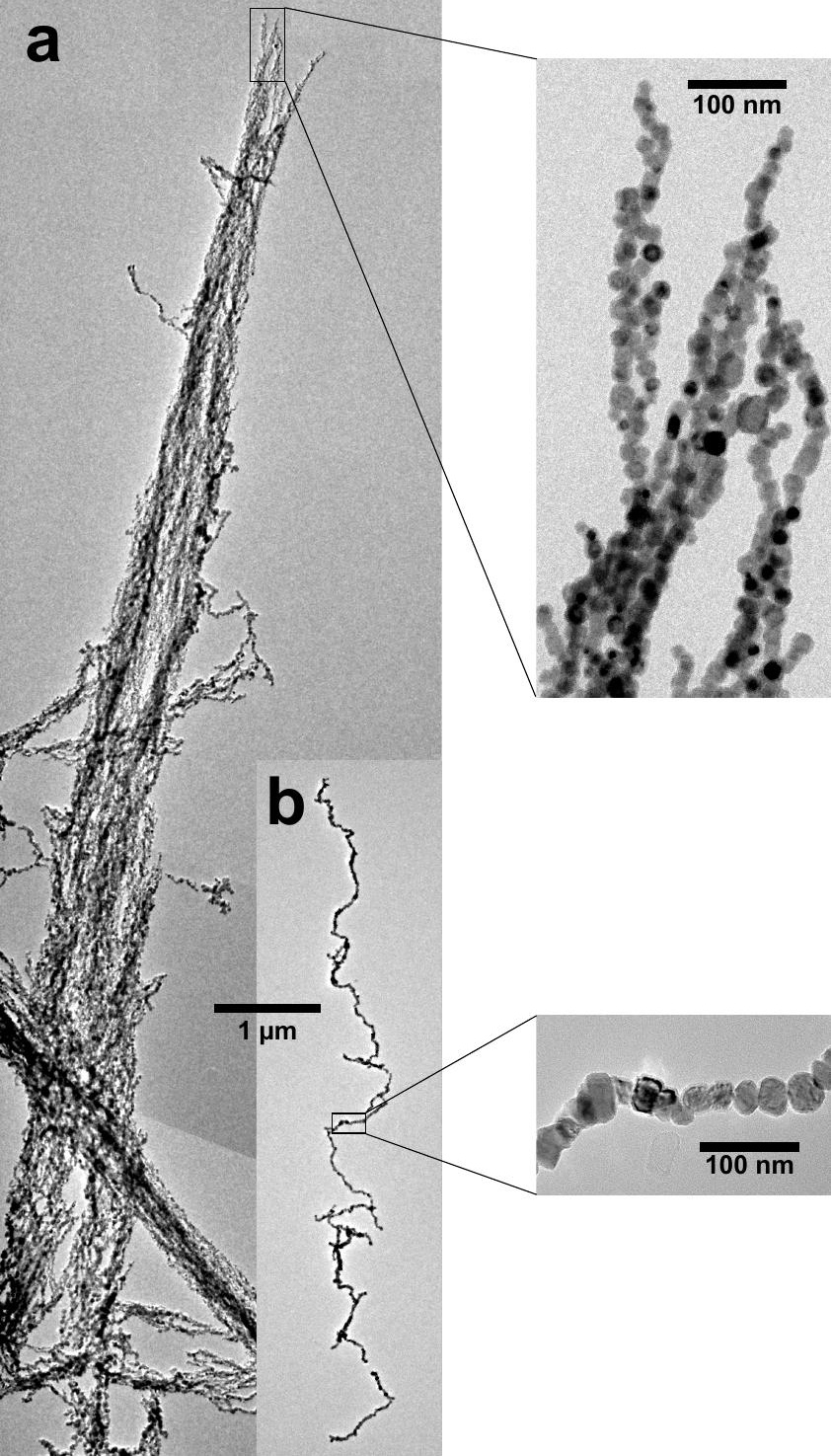




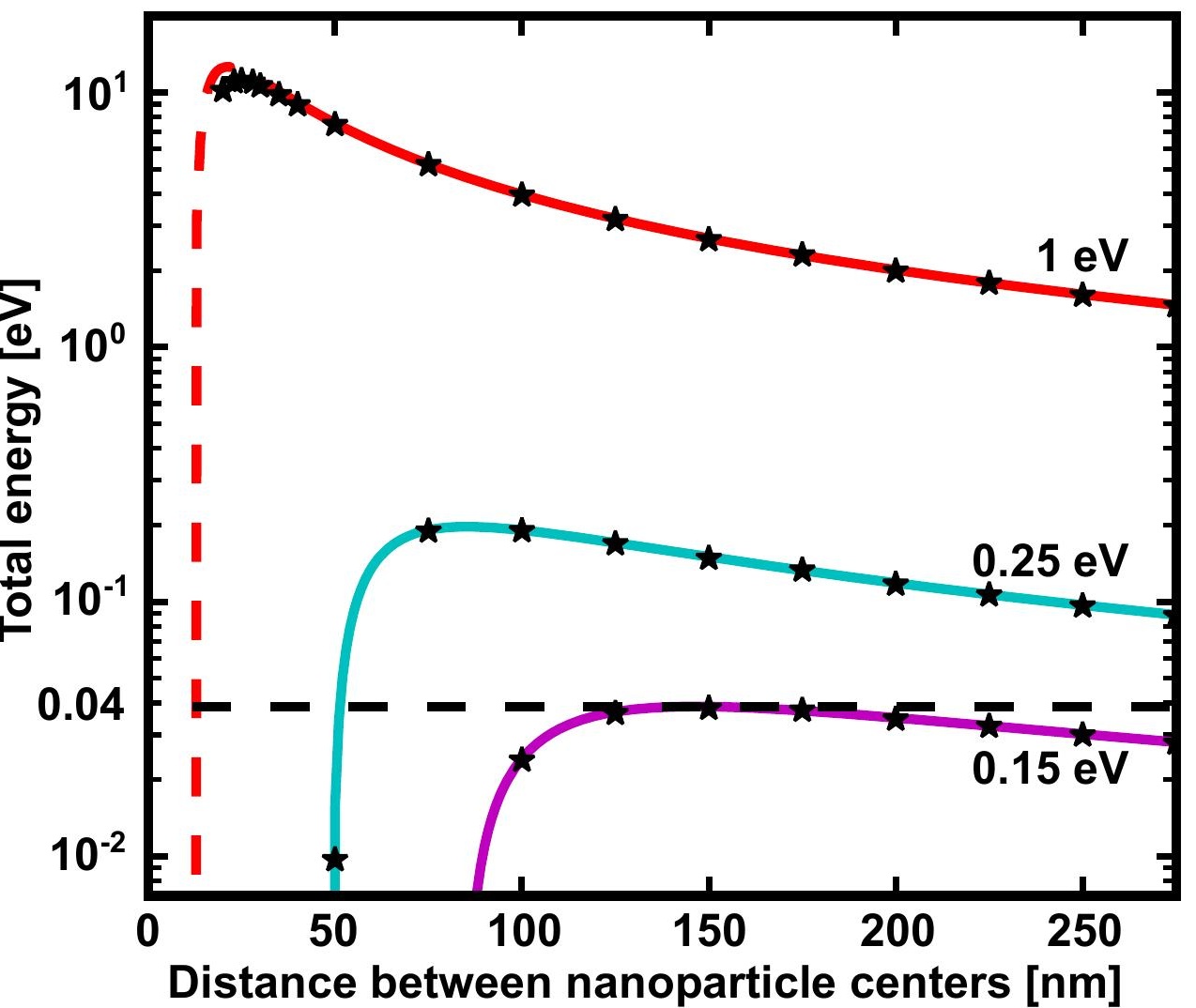




\section{Nanotrusses/ Carbon paper}

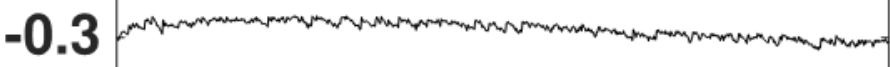

$-0.5$

0

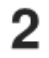

6 


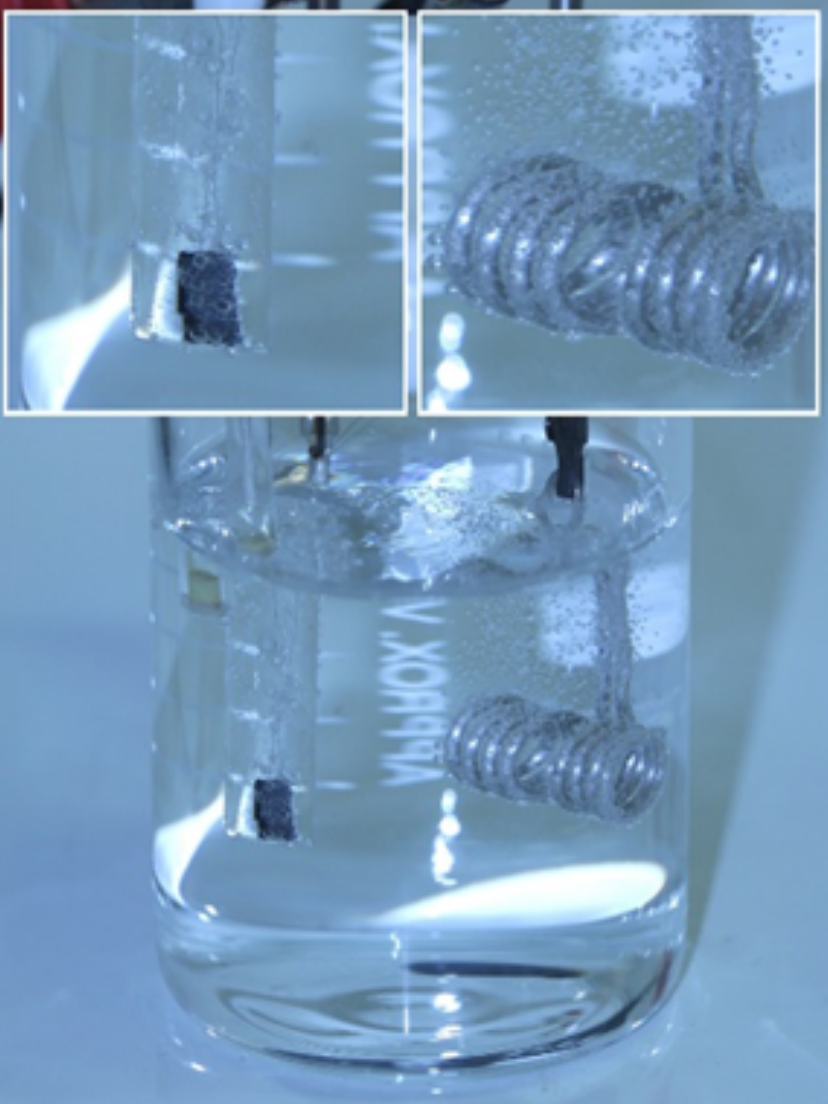




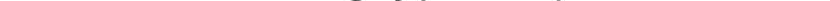

